\title{
STATIONARY HAMILTON-JACOBI EQUATIONS IN HILBERT SPACES AND APPLICATIONS TO A STOCHASTIC OPTIMAL CONTROL PROBLEM*
}

\author{
SANDRA CERRAI
}

\begin{abstract}
We study an infinite horizon stochastic control problem associated with a class of stochastic reaction-diffusion systems with coefficients having polynomial growth. The hamiltonian is assumed to be only locally Lipschitz continuous so that the quadratic case can be covered. We prove that the value function $V$ corresponding to the control problem is given by the solution of the stationary Hamilton-Jacobi equation associated with the state system. To this purpose we write the Hamilton-Jacobi equation in integral form, and, by using the smoothing properties of the transition semigroup relative to the state system and the theory of $m$-dissipative operators, we show that it admits a unique solution. Moreover, the value function $V$ is obtained as the limit of minima for some approximating control problems which admit unique optimal controls and states.
\end{abstract}

Key words. stochastic reaction-diffusion systems, stationary Hamilton-Jacobi-Bellman equations in infinite dimension, infinite horizon stochastic control problems

AMS subject classifications. 60H15, 60J35 93C20, 93E20

PII. S0363012999359949

1. Introduction. In the present paper we are concerned with an infinite horizon stochastic control problem associated with the following reaction-diffusion system perturbed by a random term:

$$
\left\{\begin{array}{l}
\frac{\partial y_{k}}{\partial t}(t, \xi)=\mathcal{A}_{k} y_{k}(t, \xi)+f_{k}\left(\xi, y_{1}(t, \xi), \ldots, y_{r}(t, \xi)\right)+z_{k}(t, \xi)+Q_{k} \frac{\partial^{2} w_{k}}{\partial t \partial \xi}(t, \xi) \\
y_{k}(0, \xi)=x_{k}(\xi), \quad t \geq 0, \quad \xi \in \overline{\mathcal{O}} \\
\mathcal{B}_{k} y_{k}(s, \xi)=0, \quad \xi \in \partial \mathcal{O}, \quad k=1, \ldots, r
\end{array}\right.
$$

Here $\mathcal{O}$ is a bounded open set in $\mathbb{R}^{d}, d \leq 3$, with regular boundary. The second order differential operators $\mathcal{A}_{k}$ are strictly elliptic, have regular coefficients, and are endowed with some boundary conditions $\mathcal{B}_{k}$. The function $f=\left(f_{1}, \ldots, f_{r}\right): \overline{\mathcal{O}} \times \mathbb{R}^{r} \rightarrow \mathbb{R}^{r}$ is twice differentiable, has polynomial growth together with its derivatives, and verifies suitable dissipativity conditions. The linear operators $Q_{k}$ are bounded and self-adjoint from $L^{2}(\mathcal{O})$ into itself and are not assumed to be Hilbert-Schmidt in general. Finally, the random fields $\partial^{2} w_{k} / \partial t \partial \xi$ are mutually independent white noises in space and in time, defined on the same stochastic basis $\left(\Omega, \mathcal{F}, \mathcal{F}_{t}, \mathbb{P}\right)$, and $z_{k}$ are square integrable processes adapted to the filtration $\mathcal{F}_{t}$.

Such a class of systems are of interest in applications and, especially in chemistry and in the present setting, have been widely studied by several authors (see, for example, Friedlin in [18] and Da Prato and Zabczyk in [14]). We recall that in [14] it

\footnotetext{
* Received by the editors August 4, 1999; accepted for publication (in revised form) February 8, 2001; published electronically September 28, 2001.

http://www.siam.org/journals/sicon/40-3/35994.html

${ }^{\dagger}$ Dipartimento di Matematica per le Decisioni, Università di Firenze, Via C. Lombroso 6/17, I-50134 Firenze, Italy (cerrai@cce.unifi.it).
} 
is proved that for any initial datum $x$ in the Hilbert space $H=L^{2}\left(\mathcal{O} ; \mathbb{R}^{r}\right)$ and for any adapted control $z \in L^{2}\left(\Omega ; L^{2}(0,+\infty ; H)\right)$ the system (1.1) admits a unique solution $y(t ; x, z)$ in a generalized sense that we will specify later. Moreover, if $x \in C\left(\overline{\mathcal{O}} ; \mathbb{R}^{r}\right)$ and $z \in L^{2}\left(\Omega ; L^{p}(0,+\infty ; H)\right)$ with $p>4 /(4-d)$, such a solution is a mild solution.

In correspondence with the system (1.1) we study the following stochastic control problem: minimizing the cost functional

$$
J(x, z)=\mathbb{E} \int_{0}^{+\infty} e^{-\lambda t}[g(y(t ; x, z))+k(z(t))] d t,
$$

among all controls $z \in L^{2}\left(\Omega ; L^{2}(0,+\infty ; H)\right)$ adapted to the filtration $\mathcal{F}_{t}$. Here $y(t ; x, z)$ is the unique solution of $(1.1)$, and $g: H \rightarrow \mathbb{R}$ is Lipschitz continuous and bounded. Moreover, $k: H \rightarrow(-\infty,+\infty]$ is a measurable mapping such that its Legendre transform $K$, which is defined by

$$
K(x)=\sup _{y \in H}\left\{-\langle x, y\rangle_{H}-k(y)\right\}, \quad x \in H,
$$

is Fréchet differentiable and locally Lipschitz continuous together with its derivative.

Our aim here is to study the value function corresponding to the functional (1.2)

$$
V(x)=\inf \left\{J(x, z) ; z \in L^{2}\left(\Omega ; L^{2}(0,+\infty ; H)\right) \text {, adapted }\right\} .
$$

Namely, we show that, if $A$ is the realization in $H$ of the differential operator $\mathcal{A}=$ $\left(\mathcal{A}_{1}, \ldots, \mathcal{A}_{r}\right)$, endowed with the boundary conditions $\mathcal{B}=\left(\mathcal{B}_{1}, \ldots, \mathcal{B}_{r}\right)$, and if $F$ is the Nemytskii operator associated with the function $f=\left(f_{1}, \ldots, f_{r}\right)$, then, under the assumption of Lipschitz continuity for $K$, for any $\lambda>0$ and $g \in C_{b}(H)^{1}$ the infinite dimensional second order nonlinear elliptic problem

$$
\lambda \varphi(x)-\frac{1}{2} \operatorname{Tr}\left[Q^{2} D^{2} \varphi(x)\right]-\langle A x+F(x), D \varphi(x)\rangle_{H}+K(D \varphi(x))=g(x)
$$

admits a unique differentiable mild solution $\varphi$. This means that there exists a unique solution $\varphi \in C_{b}^{1}(H)$ to the integral problem

$$
\varphi(x)=\mathbb{E} \int_{0}^{+\infty} e^{-\lambda t}[g(y(t ; x))-K(D \varphi(y(t ; x)))] d t,
$$

where $y(t ; x)$ is the solution of the system (1.1), corresponding to $z=0$. Moreover, for any $x \in H$ the solution $\varphi(x)$ coincides with the function $V(x)$. When $K$ is only locally Lipschitz continuous, there exists $\mu_{0}>0$ such that the same result holds for any $\lambda>\mu_{0}$ and $g \in C_{b}^{1}(H)$.

It is important to remark that even if we assume $f(\xi, \cdot)$ to be more than once differentiable, nevertheless we are able to prove only $C^{1}$-regularity in $H$ for the transition semigroup $P_{t}$ associated with the system (1.1) (see [8]). Then the solution $\varphi$ of the problem (1.3) is only $C^{1}$, and we can not prove the existence of an optimal state and an optimal control for our control problem. Actually, by following a dynamic

\footnotetext{
${ }^{1}$ We shall denote by $B_{b}(H)$ the Banach space of all bounded Borel functions $\varphi: H \rightarrow \mathbb{R}$ and by $C_{b}(H)$ the subspace of uniformly continuous functions. Moreover, we denote by $C_{b}^{k}(H), k \in \mathbb{N}$, the subspace of all $k$-times Fréchet differentiable functions, having uniformly continuous and bounded derivatives, up to the $k$ th order.
} 
programming approach, the optimal state and the optimal control would be given, respectively, by the solution $y^{\star}(t)$ of the so-called closed loop equation

$$
d y(t)=[A y(t)+F(y(t))-D K(D \varphi(y(t)))] d t+Q d w(t), \quad y(0)=x,
$$

and by

$$
z^{\star}(t)=-D K\left(D \varphi\left(y^{\star}(t)\right)\right) .
$$

On the other hand, as $D \varphi$ is only continuous, the mapping $x \mapsto-D K(D \varphi(x))$ is only continuous. Thus we are able only to prove the existence of martingale solutions for the problem (1.4), which are not defined in general in the original stochastic basis $\left(\Omega, \mathcal{F}, \mathcal{F}_{t}, \mathbb{P}\right)$, so that the control $z^{\star}(t)$ is not admissible for our original problem. However, in the case of space dimension $d=1$ it is possible to prove the existence and uniqueness of solutions for the closed loop equation and then the existence and uniqueness of an optimal control. In what follows, it could be interesting to see if, by introducing the notion of relaxed controls (see [17] for the definition and some interesting results in finite dimension), it is possible to prove the existence of an optimal control.

Nevertheless, even if we are not able to prove in general the existence of an optimal control, we can show that the value function $V$ is obtained as the limit of minima of suitable approximating cost functionals $J_{\alpha}, \alpha \geq 0$, which admit unique optimal controls and unique optimal states and whose value functions coincide with the solutions of suitable approximating Hamilton-Jacobi problems.

Several authors have studied second order Hamilton-Jacobi equations by the approach of viscosity solutions. For the finite dimensional case we refer to the paper by Crandall, Ishii, and Lions [11] and to the book by Fleming and Soner [17], and for the infinite dimensional case we refer to the papers by Lions $[25,26]$ and to the thesis of Swiech [28]. Other authors have studied regular solutions of second order HamiltonJacobi equations, and as far as the infinite dimension is concerned we refer to the works by Barbu and Da Prato [1], Cannarsa and Da Prato [2, 3], Gozzi [20, 21], Haverneau [23] for the evolution case, and by Gozzi and Rouy [22] and Chow and Menaldi [10] for the stationary case. More recently, infinite dimensional HamiltonJacobi equations have been studied in connection with some ergodic control problems (see, for example, [19] and [16]).

The main novelty here lies in the fact that we can prove the existence and uniqueness of regular solutions for (1.3) when the nonlinear coefficient $F$ in the state equation has polynomial growth and is not even well defined in the Hilbert space $H$. Moreover, we can treat both the case of a Lipschitz continuous hamiltonian and the case of a locally Lipschitz hamiltonian so that the quadratic case can be covered.

Due to the difficulties arising from coefficients which are not Lipschitz continuous, the study of mild solutions for the problem (1.3) is quite delicate, and we have to proceed in several steps. We first consider the case of a Lipschitz hamiltonian $K$, and we prove the existence and uniqueness result for $\lambda$ large enough. To this purpose, we apply a fixed point argument in the space $C_{b}^{1}(H)$, and we use the regularizing properties of the semigroup $P_{t}$ which have been studied in detail in [7] and [8]. Namely, it has been proved that

$$
\varphi \in B_{b}(H) \Longrightarrow P_{t} \varphi \in C_{b}^{1}(H), \quad t>0,
$$

and

$$
\sup _{x \in H}\left|D\left(P_{t} \varphi\right)(x)\right|_{H} \leq c(t \wedge 1)^{-\frac{1+\epsilon}{2}} \sup _{x \in H}|\varphi(x)|
$$


for some constant $\epsilon<1$ depending on $Q$. Then, if we denote by $L$ the weak generator of $P_{t}$ (see [4] for the definition and main properties) by proceeding with suitable approximations, we show that the operator

$$
N(\varphi)=L \varphi-K(D \varphi)
$$

is $m$-dissipative. This yields the existence and uniqueness of solutions for any $\lambda>0$. Then we consider a locally Lipschitz hamiltonian $K$. We approximate it by a sequence of Lipschitz functions, we consider the problems associated with the approximating hamiltonians, and, by a suitable a priori estimate, we get our result, even if in a less general case.

We remark that throughout the paper we have to proceed by several approximations because of the intrinsic difficulties in the study of the system (1.1) and because of the corresponding transition semigroup $P_{t}$. Actually, first we have to approximate the reaction term $F$ by Lipschitz continuous functionals $F_{\alpha}$ in order to get $C^{2}$ regularity for the semigroup $P_{t}^{\alpha}$ associated with the system

$$
d y(t)=\left[A y(t)+F_{\alpha}(y(t))\right] d t+Q d w(t), \quad y(0)=x,
$$

and then we have to approximate $P_{t}^{\alpha}$ by the semigroups $P_{t}^{\alpha, n}$ associated with the finite dimensional version of (1.5) in order to apply the usual Itô calculus. Unfortunately, the direct approximation of the semigroup $P_{t}$ by the semigroups $P_{t}^{\alpha, n}$ does not work.

2. Assumptions. We denote by $H$ the Hilbert space $L^{2}\left(\mathcal{O} ; \mathbb{R}^{r}\right)$, where $\mathcal{O}$ is a bounded open set of $\mathbb{R}^{d}, d \leq 3$, having the boundary sufficiently regular. The norm and the scalar product in $H$ are, respectively, denoted by $|\cdot|_{H}$ and $\langle\cdot, \cdot\rangle_{H}$. Moreover, we denote by $E$ the Banach space $C\left(\overline{\mathcal{O}} ; \mathbb{R}^{r}\right)$, endowed with the usual sup-norm $|\cdot|_{E}$.

$B_{b}(H)$ is the Banach space of bounded Borel functions $\varphi: H \rightarrow \mathbb{R}$, endowed with the sup-norm

$$
\|\varphi\|_{0}=\sup _{x \in H}|\varphi(x)| .
$$

$C_{b}(H)$ is the subspace of uniformly continuous functions. Moreover, $\operatorname{Lip}_{b}(H)$ denotes the subspace of functions $\varphi$ such that

$$
[\varphi]_{\text {Lip }}=\sup _{\substack{x, y \in H \\ x \neq y}} \frac{|\varphi(x)-\varphi(y)|}{|x-y|_{H}}<\infty .
$$

$\operatorname{Lip}_{b}(H)$ is a Banach space endowed with the norm

$$
\|\varphi\|_{\text {Lip }}=\|\varphi\|_{0}+[\varphi]_{\text {Lip }} .
$$

For each $k \in \mathbb{N}$, we denote by $C_{b}^{k}(H)$ the Banach space of $k$-times Fréchet differentiable functions, endowed with the norm

$$
\|\varphi\|_{k}=\|\varphi\|_{0}+\sum_{h=1}^{k} \sup _{x \in H}\left|D^{h} \varphi(x)\right|_{\mathcal{L}^{h}(H)} .
$$

(Here and in what follows $\mathcal{L}^{h}(H)=\mathcal{L}\left(H ; \mathcal{L}^{h-1}(H)\right), h \geq 1$, and $\mathcal{L}^{0}(H)=\mathbb{R}$.) Finally, for any $k \in \mathbb{N}$ and $\theta \in(0,1)$, we denote by $C_{b}^{k+\theta}(H)$ the subspace of all functions $\varphi \in C_{b}^{k}(H)$ such that

$$
[\varphi]_{k+\theta}=\sup _{\substack{x, y \in H \\ x \neq y}} \frac{\left|D^{k} \varphi(x)-D^{k} \varphi(y)\right|_{\mathcal{L}^{k}(H)}}{|x-y|_{H}^{\theta}}<\infty .
$$


$C_{b}^{k+\theta}(H)$ is a Banach space endowed with the norm

$$
\|\varphi\|_{k+\theta}=\|\varphi\|_{k}+[\varphi]_{k+\theta} .
$$

In what follows we shall assume that for any $\xi \in \overline{\mathcal{O}}$ and $\sigma=\left(\sigma_{1}, \ldots, \sigma_{r}\right) \in \mathbb{R}^{r}$

$$
f_{k}\left(\xi, \sigma_{1}, \ldots, \sigma_{r}\right)=g_{k}\left(\xi, \sigma_{k}\right)+h_{k}\left(\xi, \sigma_{1}, \ldots, \sigma_{r}\right), \quad k=1, \ldots, r .
$$

The functions $g_{k}: \overline{\mathcal{O}} \times \mathbb{R} \rightarrow \mathbb{R}$ and $h_{k}: \overline{\mathcal{O}} \times \mathbb{R}^{r} \rightarrow \mathbb{R}$ are continuous. Moreover, they are assumed to fulfill the following conditions.

Hypothesis 1.

1. For any $\xi \in \overline{\mathcal{O}}$, the function $h_{k}(\xi, \cdot)$ is of class $C^{2}$ and has bounded derivatives, uniformly with respect to $\xi \in \overline{\mathcal{O}}$. Moreover, the mappings $D_{\sigma}^{j} h_{k}$ : $\overline{\mathcal{O}} \times \mathbb{R}^{r} \rightarrow \mathbb{R}$ are continuous for $j=1,2$.

2. For any $\xi \in \overline{\mathcal{O}}$, the function $g_{k}(\xi, \cdot)$ is of class $C^{2}$, and there exists $m \geq 0$ such that

$$
\sup _{\xi \in \overline{\mathcal{O}}} \sup _{t \in \mathbb{R}} \frac{\left|D_{t}^{j} g_{k}(\xi, t)\right|}{1+|t|^{2 m+1-j}}<\infty
$$

Moreover, the mappings $D_{t}^{j} g_{k}: \overline{\mathcal{O}} \times \mathbb{R} \rightarrow \mathbb{R}$ are continuous for $j=1,2$.

3. If $m \geq 1$, there exist $a>0$ and $c \in \mathbb{R}$ such that

$$
\sup _{\xi \in \overline{\mathcal{O}}} D_{t} g_{k}(\xi, t) \leq-a t^{2 m}+c, \quad t \in \mathbb{R} .
$$

Notice that if $c_{k}$ and $c_{k j}$ are continuous functions from $\overline{\mathcal{O}}$ into $\mathbb{R}$ for $k=1, \ldots, r$ and $j=1, \ldots, 2 m$, and

$$
\inf _{\xi \in \overline{\mathcal{O}}} c_{k}(\xi)>0
$$

then, for any $k=1, \ldots, r$, the function

$$
g_{k}(\xi, t)=c_{k}(\xi) t^{2 m+1}+\sum_{j=1}^{2 m} c_{k j}(\xi) t^{j}
$$

fulfills the conditions of the Hypothesis 1.

Now we define the operator $F$ by setting for any function $x: \overline{\mathcal{O}} \rightarrow \mathbb{R}^{r}$

$$
F(x)(\xi)=f(\xi, x(\xi)), \quad \xi \in \overline{\mathcal{O}} .
$$

If we set $p_{\star}=2 m+2$ and $q_{\star}=(2 m+2) /(2 m+1)$, then $F$ is continuous from $L^{p_{\star}}\left(\mathcal{O} ; \mathbb{R}^{r}\right)$ into $L^{q_{\star}}\left(\mathcal{O} ; \mathbb{R}^{r}\right)$, and if $m \geq 1$, it is twice Fréchet differentiable. In particular, from (2.1) and the mean-value theorem for $x, y \in L^{p_{\star}}\left(\mathcal{O} ; \mathbb{R}^{r}\right)$, it holds that

$$
\langle F(x)-F(y), x-y\rangle_{H} \leq c|x-y|_{H}^{2} .
$$

In the same way, we have that the functional $F$ is twice differentiable and dissipative from $E$ into itself. (For more details on the properties of $F$ we refer to [7] and [9].) Notice that due to the growth conditions on $f$, the functional $F$ is not even well defined in $H$. 
As in [9], we can construct a sequence of functionals $\left\{F_{\alpha}\right\}_{\alpha}$ which are Lipschitz continuous both in $H$ and in $E$ and such that for any $x, y \in H$

$$
\left\langle F_{\alpha}(x)-F_{\alpha}(y), x-y\right\rangle_{H} \leq c|x-y|_{H}^{2}
$$

for a suitable constant $c$ independent of $\alpha>0$. Moreover, they are twice Fréchet differentiable in $E$ and for each $j \leq 2$ and $R>0$

$$
\lim _{\alpha \rightarrow 0} \sup _{|x|_{E} \leq R}\left|D^{j} F_{\alpha}(x)-D^{j} F(x)\right|_{\mathcal{L}^{j}(E)}=0 .
$$

Concerning the differential operator $\mathcal{A}=\left(\mathcal{A}_{1}, \ldots, \mathcal{A}_{r}\right)$, we assume that for any $k=1, \ldots, r$

$$
\mathcal{A}_{k}(\xi, D)=\sum_{i, j=1}^{d} a_{i j}^{k}(\xi) \frac{\partial^{2}}{\partial \xi_{i} \partial \xi_{j}}+\sum_{i=1}^{d} b_{i}^{k}(\xi) \frac{\partial}{\partial \xi_{i}}, \quad \xi \in \overline{\mathcal{O}} .
$$

The coefficients $a_{i j}^{k}$ and $b_{i}^{k}$ are of class $C^{1}(\overline{\mathcal{O}})$, and for any $\xi \in \overline{\mathcal{O}}$ the matrix $\left[a_{i j}^{k}(\xi)\right]$ is symmetric and strictly positive, uniformly with respect to $\xi \in \overline{\mathcal{O}}$. The boundary operators $\mathcal{B}_{k}$ are given by

$$
\mathcal{B}_{k}(\xi, D)=I \quad \text { or } \quad \mathcal{B}_{k}(\xi, D)=\sum_{i, j=1}^{d} a_{i j}^{k}(\xi) \nu_{j}(\xi) \frac{\partial}{\partial \xi_{i}}, \quad \xi \in \overline{\mathcal{O}}
$$

where $\nu$ is the exterior normal to the boundary of $\mathcal{O}$.

We denote by $A$ the realization in $H$ of the differential operator $\mathcal{A}$ equipped with the boundary conditions $\mathcal{B}$. The unbounded operator $A: D(A) \subset H \rightarrow H$ generates an analytic semigroup $e^{t A}$, which is not restrictive to assume of negative type. Thus we have

$$
\langle A x, x\rangle_{H} \leq 0, \quad x \in D(A) .
$$

Notice that each $L^{p}\left(\mathcal{O} ; \mathbb{R}^{r}\right), p \in[1,+\infty]$, is invariant for the semigroup $e^{t A}$, and if $p>1$, then $e^{t A}$ is analytic in $L^{p}\left(\mathcal{O} ; \mathbb{R}^{r}\right)$. Moreover, $E$ is invariant for $e^{t A}$ as well, and $e^{t A}$ generates an analytic semigroup in $E$ which is not strongly continuous. (For the proofs of these facts we refer to [15] and [27].)

Now, for any $k=1, \ldots, r$ we define

$$
\mathcal{G}_{k}(\xi, D)=\sum_{i=1}^{d}\left(b_{i}^{k}(\xi)-\sum_{j=1}^{d} \frac{\partial a_{i j}^{k}}{\partial \xi_{j}}(\xi)\right) \frac{\partial}{\partial \xi_{i}}, \quad \xi \in \overline{\mathcal{O}},
$$

and by difference we set $\mathcal{C}_{k}=\mathcal{A}_{k}-\mathcal{G}_{k}$. The realization $C$ of the operator $\mathcal{C}=$ $\left(\mathcal{C}_{1}, \ldots, \mathcal{C}_{r}\right)$ with the boundary conditions $\mathcal{B}$ generates in $H$ a self-adjoint analytic semigroup $e^{t C}$. In what follows we denote by $Q$ the bounded linear operator of components $Q_{1}, \ldots, Q_{r}$.

Hypothesis 2 .

1. There exists a complete orthonormal basis $\left\{e_{k}\right\}$ in $H$ which diagonalizes $C$ such that $\sup _{k \in \mathbb{N}}\left|e_{k}\right|_{E}<\infty$. The corresponding set of eigenvalues is denoted by $\left\{-\alpha_{k}\right\}$. 
2. The bounded linear operator $Q: H \rightarrow H$ is nonnegative and diagonal with respect to the complete orthonormal basis $\left\{e_{k}\right\}$ which diagonalizes $C$. Moreover, if $\left\{\lambda_{k}\right\}$ is the corresponding set of eigenvalues, we have

$$
\sum_{k=1}^{\infty} \frac{\lambda_{k}^{2}}{\alpha_{k}^{1-\gamma}}<+\infty
$$

for some $\gamma>0$.

3. There exists $\epsilon<1$ such that

$$
D\left((-C)^{\frac{\epsilon}{2}}\right) \subset D\left(Q^{-1}\right) .
$$

If the operator $\mathcal{A}$ with the boundary conditions $\mathcal{B}$ is smooth enough, then $\alpha_{k} \asymp$ $k^{2 / d}$. Thus, if we assume that $\lambda_{k} \asymp \alpha_{k}^{-\rho}$, when $d \leq 3$ it is possible to find some $\rho$ such that the conditions of Hypothesis 2 are verified. (For more details see [7] and [8].)

In what follows we shall denote by $P_{n}$ the projection operator of $H$ onto $H_{n}$, the subspace generated by the eigenfunctions $\left\{e_{1}, \ldots, e_{n}\right\}$. Then for any $x \in H$ we define $A_{n} x=P_{n} A P_{n} x$ and $F_{\alpha, n}(x)=P_{n}\left(F_{\alpha}\left(P_{n} x\right)\right)$. It is immediate to check that there exists a constant $c$ independent of $\alpha>0$ and $n \in \mathbb{N}$ such that

$$
\left\langle F_{\alpha, n}(x)-F_{\alpha, n}(y), x-y\right\rangle_{H} \leq c|x-y|_{H}^{2} .
$$

Next, let $\left\{w_{k}(t)\right\}$ be a sequence of mutually independent real-valued Brownian motions defined on a stochastic basis $\left(\Omega, \mathcal{F}, \mathcal{F}_{t}, \mathbb{P}\right)$. The cylindrical Wiener process $w(t)$ is formally defined as

$$
\sum_{k=1}^{\infty} e_{k} w_{k}(t)
$$

where $\left\{e_{k}\right\}$ is the orthonormal basis of $H$ introduced in Hypothesis 2(1). Under the Hypotheses 2(1) and 2(2) it is possible to show that the linear problem associated with the system (1.1),

$$
d z(t)=A z(t) d t+Q d w(t), \quad z(0)=0,
$$

admits a unique solution $w^{A}(t)$ which is the mean-square Gaussian process with values in $H$ given by

$$
w^{A}(t)=\int_{0}^{t} e^{(t-s) A} Q d w(s) .
$$

As shown, for example, in [14], the process $w^{A}$ belongs to $C([0,+\infty) \times \overline{\mathcal{O}}), \mathbb{P}$ almost surely (a.s), and for any $p \geq 1$ and $T>0$ it holds that

$$
\mathbb{E} \sup _{t \in[0, T]}\left|w^{A}\right|_{E}^{p}<\infty
$$

3. The transition semigroup. By using the notations introduced in the previous section, the system (1.1) can be rewritten as

$$
d y(t)=[A y(t)+F(y(t))+z(t)] d t+Q d w(t), \quad y(0)=x .
$$

The following theorem is proved in [14] in the uncontrolled case. The proof in the controlled case is analogous; thus we omit it. (For more details we refer also to [7] and [8].) 
Theorem 3.1. Assume Hypotheses 1 and 2.

1. For any $x \in E$ and for any adapted process $z \in L^{2}\left(\Omega ; L^{p}(0,+\infty ; H)\right)$ with $p>4 /(4-d)$, there exists a unique mild solution $y(\cdot ; x, z)$ for the problem $(3.1)$ which belongs to $L^{2}\left(\Omega ; C((0, T] ; E) \cap L^{\infty}(0, T ; E)\right)$, for any $T>0$. This means that

$$
y(t ; x, z)=e^{t A} x+\int_{0}^{t} e^{(t-s) A} F(y(s ; x, z)) d s+w^{A}(t),
$$

where $w^{A}(t)$ is the solution of the linear system (2.6). Moreover, it holds that

$$
|y(t ; x, z)|_{E} \leq c(t)\left(|x|_{E}+|z|_{L^{p}(0,+\infty ; H)}^{2 m+1}+\sup _{s \in[0, t]}\left|w^{A}(s)\right|_{E}^{2 m+1}\right),
$$

$\mathbb{P}$-a.s. for a suitable continuous increasing function $c(t)$.

2. For any $x \in H$ and for any adapted process $z \in L^{2}\left(\Omega ; L^{2}(0,+\infty ; H)\right)$, there exists a unique generalized solution $y(\cdot ; x, z) \in L^{2}(\Omega ; C([0,+\infty) ; H))$ for the problem (3.1). This means that for any sequence $\left\{z_{n}\right\} \subset L^{2}\left(\Omega ; L^{p}(0,+\infty ; H)\right)$ converging to $z$ in $L^{2}\left(\Omega ; L^{2}(0,+\infty ; H)\right)$ and for any sequence $\left\{x_{n}\right\} \subset E$ converging to $x$ in $H$, the corresponding sequence of mild solutions $\left\{y\left(\cdot ; x_{n}, z_{n}\right)\right\}$ converges to the process $y(\cdot ; x, z)$ in $C([0, T] ; H), \mathbb{P}$-a.s., for any fixed $T>0$. Moreover, it holds that

$$
|y(t ; x, z)|_{H} \leq c(t)\left(|x|_{H}+|z|_{L^{2}(0,+\infty ; H)}^{2 m+1}+\sup _{s \in[0, t]}\left|w^{A}(s)\right|_{E}^{2 m+1}\right),
$$

$\mathbb{P}$-a.s., for a suitable continuous increasing function $c(t)$.

3. The generalized solution $y(\cdot ; x, z)$ belongs to $L^{2 m+2}\left(0,+\infty ; L^{2 m+2}\left(\mathcal{O} ; \mathbb{R}^{r}\right)\right)$, $\mathbb{P}$-a.s., and fulfills the integral equation (3.2).

4. For any $x_{1}, x_{2} \in H$ and $z_{1}, z_{2} \in L^{2}\left(\Omega ; L^{2}(0,+\infty ; H)\right)$ it holds that

$$
\left|y\left(t ; x_{1}, z_{1}\right)-y\left(t ; x_{2}, z_{2}\right)\right|_{H} \leq c(t)\left(\left|x_{1}-x_{2}\right|_{H}+\left|z_{1}-z_{2}\right|_{L^{2}(0, t ; H)}\right),
$$

$\mathbb{P}$-a.s., for a suitable continuous increasing function $c(t)$.

Next, for any $\alpha>0$, we introduce the approximating problem

$$
d y(t)=\left(A y(t)+F_{\alpha}(y(t))+z(t)\right) d t+Q d w(t), \quad y(0)=x .
$$

If $x \in H$ and $z \in L^{2}\left(\Omega ; L^{2}(0,+\infty ; H)\right)$, the system (3.5) admits a unique mild solution $y_{\alpha}(\cdot ; x, z) \in L^{2}(\Omega ; C([0,+\infty) ; H))$. If $x \in E$ and $z \in L^{2}\left(\Omega ; L^{p}(0,+\infty ; H)\right)$ with $p>4 /(4-d)$, then $y_{\alpha}(\cdot ; x, z) \in L^{2}\left(\Omega ; C((0, T] ; E) \cap L^{\infty}(0, T ; E)\right)$ for any $T>0$. Moreover, an estimate analogous to (3.3) holds, uniformly with respect to $\alpha>0$. Namely, there exists an increasing continuous function $c(t)$ independent of $\alpha$ such that

$$
\left|y_{\alpha}(t ; x, z)\right|_{E} \leq c(t)\left(|x|_{E}+|z|_{L^{p}(0,+\infty ; H)}^{2 m+1}+\sup _{s \in[0, t]}\left|w^{A}(s)\right|_{E}^{2 m+1}\right),
$$

$\mathbb{P}$-a.s. The following approximation result has been proved already in [9].

Proposition 3.2. Under the Hypotheses 1 and 2, for any $q \geq 1$ there exists $p \geq 1$ such that if $x \in E$ and $z \in L^{p}\left(\Omega ; L^{\infty}(0,+\infty ; H)\right)$, then it holds that

$$
\lim _{\alpha \rightarrow 0} \mathbb{E}\left|y(t ; x, z)-y_{\alpha}(t ; x, z)\right|_{E}^{q}=0, \quad \mathbb{P} \text {-a.s. }
$$


uniformly with respect to $(t, x)$ in bounded sets of $[0,+\infty) \times E$ and $z$ in the set

$$
\mathcal{M}_{R}^{2}=\left\{z \in L^{2}\left(\Omega ; L^{2}(0,+\infty ; H)\right): \sup _{t \geq 0}|z(t)|_{H} \leq R, \quad \mathbb{P} \text {-a.s. }\right\}
$$

for any $R \geq 0$.

For any $\alpha>0$ and $n \in \mathbb{N}$, we denote by $y_{\alpha, n}(\cdot ; x, z)$ the unique strong solution in $L^{2}(\Omega ; C([0,+\infty) ; H))$ of the approximating problem

$$
d y(t)=\left(A_{n} y(t)+F_{\alpha, n}(y(t))+P_{n} z(t)\right) d t+Q_{n} d w(t), \quad y(0)=P_{n} x,
$$

with $x \in H$ and $z \in L^{2}\left(\Omega ; L^{2}(0,+\infty ; H)\right)$ adapted. In [9, Lemma 3.4] we have shown that for any fixed $R, T>0$

$$
\lim _{n \rightarrow+\infty} \sup _{|x|_{H} \leq R}\left|y_{\alpha, n}(\cdot ; x, z)-y_{\alpha}(\cdot ; x, z)\right|_{L^{2}(\Omega ; C([0, T] ; H))}=0 .
$$

Moreover, we have

$$
\left|y_{\alpha, n}(t ; x, z)\right|_{H} \leq c(t)\left(|x|_{H}+|z|_{L^{2}(0,+\infty ; H)}^{2 m+1}+\sup _{s \in[0, t]}\left|w^{A}(s)\right|_{E}^{2 m+1}\right), \quad \mathbb{P} \text {-a.s. }
$$

In what follows we shall denote by $y(t ; x)$ the solution of $(3.1)$ with $z=0$. In [7, Theorem 7.4] we have shown that if $f(\xi, \cdot)$ is $k$-times differentiable, then for any $t \geq 0$ the mapping

$$
E \rightarrow L^{2}(\Omega ; E), \quad x \mapsto y(t ; x)
$$

is $k$-times Fréchet differentiable. In particular, the first derivative $D y(t ; x) h$ is the unique solution of the linearized problem

$$
\frac{d v}{d t}(t)=A v(t)+D F(y(t ; x)) v(t), \quad v(0)=h,
$$

and it holds that

$$
\sup _{x \in E}|D y(t ; x) h|_{H} \leq e^{c t}|h|_{H}, \quad \mathbb{P} \text {-a.s. }
$$

If $x, h \in H$, then, as shown in [8], the problem above admits a unique generalized solution $v(t ; x, h)$ which is not intended to be the mean-square derivative of $y(t ; x)$ in general.

In [6] we have proved that, since $F_{\alpha}$ and $F_{\alpha, n}$ are Lipschitz continuous, $y_{\alpha}(t ; x)$ and $y_{\alpha, n}(t ; x)$ are twice mean-square differentiable with respect to $x \in H$ along any direction $h \in H$. In addition, their derivatives belong to $D\left(A^{1 / 2}\right) \subset D\left(Q^{-1}\right)$ and for any $T>0$

$$
\begin{aligned}
& \sup _{x \in H}\left|D y_{\alpha}(\cdot ; x) h\right|_{L^{\infty}(0, T ; H) \cap L^{2}\left(0, T ; D\left(A^{1 / 2}\right)\right)} \leq c_{T}|h|_{H}, \quad \mathbb{P} \text {-a.s., } \\
& \sup _{x \in H}\left|D y_{\alpha, n}(t ; x) h\right|_{L^{\infty}(0, T ; H) \cap L^{2}\left(0, T ; D\left(A^{1 / 2}\right)\right)} \leq c_{T}|h|_{H}, \quad \mathbb{P} \text {-a.s., }
\end{aligned}
$$


for a constant $c_{T}$ which is independent of $\alpha>0$ and $n \in \mathbb{N}$. In [9, Lemma 4.1] we have also proved that

$$
\lim _{\alpha \rightarrow 0} \mathbb{E} \sup _{|h|_{H} \leq 1}\left|D y(\cdot ; x) h-D y_{\alpha}(\cdot ; x) h\right|_{L^{\infty}(0, T ; H) \cap L^{2}\left(0, T ; D\left((-A)^{1 / 2}\right)\right)}^{2}=0,
$$

uniformly with respect to $x$ in bounded sets of $E$, and in [9, Lemma 4.2] we have proved that

$$
\lim _{n \rightarrow+\infty} \mathbb{E} \sup _{|h|_{H} \leq 1}\left|D y_{\alpha}(\cdot ; x) h-D y_{\alpha, n}(\cdot ; x) h\right|_{L^{\infty}(0, T ; H) \cap L^{2}\left(0, T ; D\left((-A)^{1 / 2}\right)\right)}^{2}=0,
$$

uniformly with respect to $x$ in bounded sets of $H$.

Next we define the transition semigroup $P_{t}$ corresponding to the system (3.1) by setting for any $\varphi \in B_{b}(H)$ and $x \in H$

$$
P_{t} \varphi(x)=\mathbb{E} \varphi(y(t ; x)), \quad t \geq 0 .
$$

In an analogous way, we define the semigroups $P_{t}^{\alpha}$ and $P_{t}^{\alpha, n}$ associated, respectively, to the systems (3.5) and (3.9). Due to (3.7), for any $\varphi \in C_{b}(H)$ and $R>0$

$$
\lim _{\alpha \rightarrow 0} \sup _{|x|_{E} \leq R}\left|P_{t}^{\alpha} \varphi(x)-P_{t} \varphi(x)\right|=0,
$$

uniformly for $t$ in bounded sets of $[0,+\infty)$. Moreover, due to (3.10) we have that

$$
\lim _{n \rightarrow+\infty} \sup _{|x|_{H} \leq R}\left|P_{t}^{\alpha, n} \varphi(x)-P_{t}^{\alpha} \varphi(x)\right|=0,
$$

uniformly for $t$ in bounded sets of $[0,+\infty)$. It is important to notice that all the properties of the semigroup $P_{t}$ which we are going to describe are fulfilled by the semigroups $P_{t}^{\alpha}$ and $P_{t}^{\alpha, n}$ as well.

From (3.4) it easily follows that $P_{t}$ maps $C_{b}(H)$ into itself as a contraction. In general $P_{t}$ is not strongly continuous in $C_{b}(H)$. (See [4] for a counter example even in finite dimension.) Nevertheless, as $y(\cdot ; x) \in L^{2}(\Omega ; C([0,+\infty) ; H))$ for any fixed $x \in H$, by the dominated convergence theorem, we have that if $\varphi \in C_{b}(H)$, then the mapping

$$
[0,+\infty) \rightarrow \mathbb{R}, \quad t \mapsto P_{t} \varphi(x)
$$

is continuous. Thus, by proceeding as in [4], we define the generator $L$ of $P_{t}$ as the unique closed operator $L: D(L) \subset C_{b}(H) \rightarrow C_{b}(H)$ such that

$$
R(\lambda, L) \varphi(x)=\int_{0}^{+\infty} e^{-\lambda t} P_{t} \varphi(x) d t, \quad \lambda>0,
$$

for any fixed $\varphi \in C_{b}(H)$ and $x \in H$. In a similar way we define the generators $L_{\alpha}$ and $L_{\alpha, n}$ corresponding, respectively, to the semigroups $P_{t}^{\alpha}$ and $P_{t}^{\alpha, n}$.

In [4] it is shown that for any $\varphi \in D(L)$ and $x \in H$ the mapping

$$
[0,+\infty) \rightarrow \mathbb{R}, \quad t \mapsto P_{t} \varphi(x)
$$

is differentiable and

$$
\frac{d}{d t} P_{t} \varphi(x)=L\left(P_{t} \varphi\right)(x)=P_{t}(L \varphi)(x) .
$$


The same holds for $L_{\alpha}$ and $L_{\alpha, n}$. In particular, if $\varphi \in C_{b}^{2}(H)$, we have that $P_{s}^{\alpha, n} \varphi \in$ $D\left(L_{\alpha, n}\right)$, for any $\alpha>0, n \in \mathbb{N}$, and $s \geq 0$, and

$$
L_{\alpha, n}\left(P_{s}^{\alpha, n} \varphi\right)=\mathcal{L}_{\alpha, n}\left(P_{s}^{\alpha, n} \varphi\right),
$$

where the differential operator $\mathcal{L}_{\alpha, n}$ is defined by

$$
\mathcal{L}_{\alpha, n} \varphi(x)=\frac{1}{2} \operatorname{Tr}\left[Q_{n}^{2} D^{2} \varphi(x)\right]+\left\langle A_{n} x+F_{\alpha, n}(x), D \varphi(x)\right\rangle_{H}, \quad x \in H .
$$

Actually, if we define $\psi=\lambda P_{s}^{\alpha, n} \varphi-\mathcal{L}_{\alpha, n}\left(P_{s}^{\alpha, n} \varphi\right)$ for some $\lambda>0$, we have that

$$
R\left(\lambda, L_{\alpha, n}\right) \psi(x)=\int_{0}^{+\infty} e^{-\lambda t}\left[\lambda P_{t+s}^{\alpha, n} \varphi(x)-P_{t}^{\alpha, n} \mathcal{L}_{\alpha, n}\left(P_{s}^{\alpha, n} \varphi\right)(x)\right] d t .
$$

It is not difficult to prove that, in general, if $\varphi$ is twice differentiable, then

$$
P_{t}^{\alpha, n}\left(\mathcal{L}_{\alpha, n} \varphi\right)(x)=\mathcal{L}_{\alpha, n}\left(P_{t}^{\alpha, n} \varphi\right)(x) .
$$

Thus, as $P_{s}^{\alpha, n} \varphi \in C_{b}^{2}(H)$, from the Itô formula we have

$$
P_{t}^{\alpha, n} \mathcal{L}_{\alpha, n}\left(P_{s}^{\alpha, n} \varphi\right)(x)=\mathcal{L}_{\alpha, n}\left(P_{t+s}^{\alpha, n} \varphi\right)(x)=\frac{d}{d t}\left(P_{t+s}^{\alpha, n} \varphi(x)\right) .
$$

This allows us to conclude that

$$
R\left(\lambda, L_{\alpha, n}\right) \psi(x)=-\int_{0}^{+\infty} \frac{d}{d t}\left(e^{-\lambda t} P_{t+s}^{\alpha, n} \varphi(x)\right) d t=P_{s}^{\alpha, n} \varphi(x),
$$

so that $P_{s}^{\alpha, n} \varphi \in D\left(L_{\alpha, n}\right)$ and (3.15) holds.

In [8] we have proved that the semigroup $P_{t}$ has a smoothing effect. Namely, it maps $B_{b}(H)$ into $C_{b}^{1}(H)$ for any $t>0$, and for $i \leq j=0,1$ it holds that

$$
\left\|P_{t} \varphi\right\|_{j} \leq c(t \wedge 1)^{-\frac{(j-i)(1+\epsilon)}{2}}\|\varphi\|_{i}
$$

where $\epsilon$ is the constant introduced in Hypothesis 2(3). As far as the semigroups $P_{t}^{\alpha}$ and $P_{t}^{\alpha, n}$ are concerned, in [6] it is proved that they map $B_{b}(H)$ into $C_{b}^{2}(H)$ for any $t>0$, and

$$
\left\|P_{t}^{\alpha} \varphi\right\|_{j}+\left\|P_{t}^{\alpha, n} \varphi\right\|_{j} \leq c_{\alpha}(t \wedge 1)^{-\frac{(j-i)(1+\epsilon)}{2}}\|\varphi\|_{i}
$$

for any $i \leq j \leq 2$, for some constant $c_{\alpha}$ independent of $n$. Moreover, if $i \leq j \leq 1$, the constant $c_{\alpha}$ is independent of $\alpha$ as well.

We conclude, recalling that in [9] it has been proved that if $\varphi \in C_{b}(H)$, then for any $R>0$

$$
\lim _{\alpha \rightarrow 0} \sup _{|x|_{E} \leq R}\left|D\left(P_{t}^{\alpha} \varphi\right)(x)-D\left(P_{t} \varphi\right)(x)\right|_{H}=0,
$$

uniformly for $t$ in bounded sets of $[\delta,+\infty)$ with $\delta>0$. Moreover, it has been proved that

$$
\lim _{n \rightarrow+\infty} \sup _{|x|_{H} \leq R}\left|D\left(P_{t}^{\alpha, n} \varphi\right)(x)-D\left(P_{t}^{\alpha} \varphi\right)(x)\right|_{H}=0,
$$

uniformly for $t$ in bounded sets of $[\delta,+\infty)$ with $\delta>0$. 
4. The Hamilton-Jacobi equation. We are here concerned with the stationary Hamilton-Jacobi equation

$$
\lambda \varphi(x)-L \varphi(x)+K(D \varphi(x))=g(x), \quad x \in H .
$$

Our aim is to show that such an equation admits a unique solution $\varphi(\lambda, g)$ for any $\lambda>0$ and $g \in C_{b}(H)$. To this purpose we first prove a regularity result for the elements of $D(L)$.

Lemma 4.1. Assume Hypotheses 1 and 2. Then $D(L) \subset C_{b}^{1}(H)$, and for any $\lambda>0$ and $g \in C_{b}(H)$ it holds that

$$
\|R(\lambda, L) g\|_{1} \leq \rho(\lambda)\|g\|_{0}
$$

where $\rho(\lambda)=c\left(\lambda^{\frac{\epsilon-1}{2}}+\lambda^{-1}\right)$.

Proof. We recall that if $\varphi \in C_{b}(H)$, then $P_{t} \varphi \in C_{b}^{1}(H)$ for any $t>0$. Thus for any $x, h \in H$ and $\lambda>0$ we have

$$
\begin{aligned}
& R(\lambda, L) g(x+h)-R(\lambda, L) g(x)=\int_{0}^{+\infty} e^{-\lambda t}\left(P_{t} g(x+h)-P_{t} g(x)\right) d t \\
& =\int_{0}^{+\infty} e^{-\lambda t}\left\langle D\left(P_{t} g\right)(x), h\right\rangle_{H} d t+E(x, h),
\end{aligned}
$$

where

$$
E(x, h)=\int_{0}^{+\infty} e^{-\lambda t} \int_{0}^{1}\left\langle D\left(P_{t} g\right)(x+\theta h)-D\left(P_{t} g\right)(x), h\right\rangle_{H} d \theta d t .
$$

Due to (3.17) we have

$$
\begin{aligned}
& \left|\int_{0}^{+\infty} e^{-\lambda t}\left\langle D\left(P_{t} g\right)(x), h\right\rangle_{H} d t\right| \\
& \leq c \int_{0}^{+\infty} e^{-\lambda t}(t \wedge 1)^{-\frac{1+\epsilon}{2}} d t|h|_{H}\|g\|_{0}=c\left(\lambda^{\frac{\epsilon-1}{2}}+\lambda^{-1}\right)|h|_{H}\|g\|_{0} .
\end{aligned}
$$

Moreover, as $D\left(P_{t} g\right)$ is continuous in $H$, by the dominated convergence theorem we easily have that

$$
\lim _{|h|_{H} \rightarrow 0} \frac{|E(x, h)|}{|h|_{H}}=0 .
$$

This implies that $R(\lambda, L) g \in C_{b}^{1}(H)$, and for any $x, h \in H$

$$
\langle D(R(\lambda, L) g)(x), h\rangle_{H}=\int_{0}^{+\infty} e^{-\lambda t}\left\langle D\left(P_{t} g\right)(x), h\right\rangle_{H} d t
$$

so that the estimate (4.2) holds true.

Remark 4.2. Notice that due to (3.18) we can repeat the arguments used above, and we can show that both $D\left(L_{\alpha}\right)$ and $D\left(L_{\alpha, n}\right)$ are contained in $C_{b}^{1}(H)$, and a formula analogous to (4.3) holds for the derivatives of $R\left(\lambda, L_{\alpha}\right) g$ and $R\left(\lambda, L_{\alpha, n}\right) g$ when $g \in C_{b}(H)$. In particular, it holds that

$$
\left\|R\left(\lambda, L_{\alpha}\right) g\right\|_{1}+\left\|R\left(\lambda, L_{\alpha, n}\right) g\right\|_{1} \leq \rho(\lambda)\|g\|_{0} .
$$


Moreover, as

$$
\left\|P_{t}^{\alpha} \varphi\right\|_{i}+\left\|P_{t}^{\alpha, n} \varphi\right\|_{i} \leq c_{\alpha}(t \wedge 1)^{-\frac{(i-j)(1+\epsilon)}{2}}\|\varphi\|_{j}, \quad j \leq i \leq 2,
$$

for a constant $c_{\alpha}$ independent of $n \in \mathbb{N}$, by interpolation we have that for any $\theta_{1}, \theta_{2} \in$ $[0,1]$

$$
\left\|P_{t}^{\alpha} \varphi\right\|_{1+\theta_{1}}+\left\|P_{t}^{\alpha, n} \varphi\right\|_{1+\theta_{1}} \leq c_{\alpha}(t \wedge 1)^{-\frac{\left(\theta_{1}-\theta_{2}+1\right)(1+\epsilon)}{2}}\|\varphi\|_{\theta_{2}} .
$$

By proceeding as in the proof of the previous lemma, this implies that if $\varphi \in C_{b}^{\theta_{2}}(H)$, then $R\left(\lambda, L_{\alpha}\right) \varphi$ and $R\left(\lambda, L_{\alpha, n}\right) \varphi$ are in $C_{b}^{1+\theta_{1}}(H)$ for any $\theta_{1}<\theta_{2}+(1-\epsilon) /(1+\epsilon)$ and

$$
\left\|R\left(\lambda, L_{\alpha}\right) \varphi\right\|_{1+\theta_{1}}+\left\|R\left(\lambda, L_{\alpha, n}\right) \varphi\right\|_{1+\theta_{1}} \leq c_{\alpha}\left(\lambda^{\frac{\left(\theta_{1}-\theta_{2}+1\right)(\epsilon+1)}{2}-1}+\lambda^{-1}\right)\|g\|_{\theta_{2}} .
$$

In particular, we have that $D\left(L_{\alpha}\right)$ and $D\left(L_{\alpha, n}\right)$ are contained in $C_{b}^{1+\theta}(H)$ for any $\theta<(1-\epsilon) /(\epsilon+1)$.

4.1. Lipschitz hamiltonian $\boldsymbol{K}$. In the proof of the existence and uniqueness of solutions for the problem (4.1) we proceed in several steps. First we assume the Lipschitz continuity of the hamiltonian $K$.

Hypothesis 3. The mapping $K: H \rightarrow \mathbb{R}$ is Fréchet differentiable and Lipschitz continuous together with its derivative. Moreover, $K(0)=0$.

Notice that the condition $K(0)=0$ is not restrictive, as we can substitute $g$ by $g-K(0)$.

By using the Lemma 4.1 we get the following result.

Proposition 4.3. Under Hypotheses 1, 2, and 3, there exists $\lambda_{0}>0$ such that (4.1) admits a unique solution $\varphi(\lambda, g) \in C_{b}^{1}(H)$ for any $\lambda>\lambda_{0}$ and for any $g \in C_{b}(H)$.

Proof. The equation (4.1) is equivalent to the equation

$$
\varphi=R(\lambda, L)(g-K(D \varphi))=\Gamma(\lambda, g)(\varphi) .
$$

Due to Lemma 4.1, if $\varphi \in C_{b}^{1}(H)$ and $g \in C_{b}(H)$, then $\Gamma(\lambda, g)(\varphi) \in C_{b}^{1}(H)$. Thus if we show that for some $\lambda_{0}>0$ the mapping $\Gamma(\lambda, g)$ is a contraction in $C_{b}^{1}(H)$ for any $\lambda>\lambda_{0}$, our thesis follows.

As $K$ is Lipschitz continuous for any $\varphi_{1}, \varphi_{2} \in C_{b}^{1}(H)$, we have

$$
\left\|R(\lambda, L)\left(K\left(D \varphi_{1}\right)-K\left(D \varphi_{2}\right)\right)\right\|_{1} \leq c \rho(\lambda)\left\|\varphi_{1}-\varphi_{2}\right\|_{1} .
$$

Thus, if we choose $\lambda_{0}$ such that $c \rho\left(\lambda_{0}\right)=1$, we have that $\Gamma(\lambda, g)$ is a contraction in $C_{b}^{1}(H)$ for any $\lambda>\lambda_{0}$. This implies that it admits a unique fixed point $\varphi \in C_{b}^{1}(H)$, which is the unique solution of (4.1) in $C_{b}^{1}(H)$.

Remark 4.4. By using (4.4) it is possible to prove that there exists $\lambda_{0}>0$ sufficiently large such that the mappings

$$
\Gamma_{\alpha}(\lambda, g)(\varphi)=R\left(\lambda, L_{\alpha}\right)(g-K(D \varphi)), \quad \alpha>0,
$$

are contractions in $C_{b}^{1}(H)$ for any $\lambda>\lambda_{0}$ and for any $g \in C_{b}(H)$, and the approximating Hamilton-Jacobi equations

$$
\lambda \varphi-L_{\alpha} \varphi+K(D \varphi)=g
$$


admit a unique solution $\varphi_{\alpha}(\lambda, g) \in C_{b}^{1}(H)$. Moreover, as the function $\rho(\lambda)$ in (4.4) does not depend on $\alpha>0$, the constant $\lambda_{0}$ does not depend on $\alpha$ either.

Lemma 4.5. Under Hypotheses 1, 2, and 3, for any $\lambda>0$ and $g \in C_{b}(H)$ we have

$$
\lim _{\alpha \rightarrow 0} \sup _{|x|_{E} \leq R}\left|D^{j}\left(\Gamma_{\alpha}^{k}(\lambda, g)(0)-\Gamma^{k}(\lambda, g)(0)\right)(x)\right|_{\mathcal{L}^{j}(H)}=0, \quad j=0,1,
$$

for any $k \in \mathbb{N}$ and $R>0$.

Proof. We proceed by induction. For $k=1$ the limit (4.7) is trivially verified. Assume that (4.7) holds for some $k \geq 1$. We show that this implies that (4.7) holds for $k+1$. We have

$$
\begin{aligned}
& D^{j}\left(\Gamma_{\alpha}^{k+1}(\lambda, g)(0)-\Gamma^{k+1}(\lambda, g)(0)\right) \\
& =D^{j}\left(R\left(\lambda, L_{\alpha}\right)\left[g-K\left(D\left(\Gamma_{\alpha}^{k}(\lambda, g)(0)\right)\right)\right]-R(\lambda, L)\left[g-K\left(D\left(\Gamma^{k}(\lambda, g)(0)\right)\right)\right]\right) .
\end{aligned}
$$

In general, if $f \in C_{b}(H)$ and $\left\{f_{\alpha}\right\}$ is any bounded generalized sequence of $C_{b}(H)$ such that for any $R>0$

$$
\lim _{\alpha \rightarrow 0} \sup _{|x|_{E} \leq R}\left|f_{\alpha}(x)-f(x)\right|=0,
$$

then for any $R>0$ and $j=0,1$ we have

$$
\lim _{\alpha \rightarrow 0} \sup _{|x|_{E} \leq R}\left|D^{j}\left(R\left(\lambda, L_{\alpha}\right)\left(f_{\alpha}-f\right)\right)(x)\right|_{H}=0 .
$$

Indeed, as the formula (4.3) holds for the derivative of $R\left(\lambda, L_{\alpha}\right)$, as well, for any $x \in H$ we have

$$
D^{j}\left(R\left(\lambda, L_{\alpha}\right)\left(f_{\alpha}-f\right)\right)(x)=\int_{0}^{+\infty} e^{-\lambda t} D^{j}\left(P_{t}^{\alpha}\left(f_{\alpha}-f\right)\right)(x) d t .
$$

If $x$ lies in a bounded set of $E$, due to $(2.7)$ and (3.6) the solution $y_{\alpha}(t ; x)(\omega)$ lies in a bounded set of $E$ for $\mathbb{P}$-almost all $\omega \in \Omega$. Therefore, by (4.8) for any $R>0$ this yields

$$
\lim _{\alpha \rightarrow 0} \sup _{|x|_{E} \leq R}\left|\left(f_{\alpha}-f\right)\left(y_{\alpha}(t ; x)\right)\right|=0, \quad \mathbb{P} \text {-a.s., }
$$

and by applying the dominated convergence theorem we get (4.9) for $j=0$. As proved in [5], for any $t>0$ we have

$$
\left\langle D\left(P_{t}^{\alpha}\left(f_{\alpha}-f\right)\right)(x), h\right\rangle_{H}=\frac{1}{t} \mathbb{E}\left(f_{\alpha}-f\right)\left(y_{\alpha}(t ; x)\right) \int_{0}^{t}\left\langle Q^{-1} D y_{\alpha}(s ; x) h, d w(s)\right\rangle_{H},
$$

where $D y_{\alpha}(t ; x) h$ is the mean-square derivative of $y_{\alpha}(t ; x)$ along the direction $h \in H$. Hence, thanks to (3.12), by interpolation we easily get

$$
\left|D\left(P_{t}^{\alpha}\left(f_{\alpha}-f\right)\right)(x)\right|_{H} \leq c(t \wedge 1)^{-\frac{1+\epsilon}{2}}\left(\mathbb{E}\left|\left(f_{\alpha}-f\right)\left(y_{\alpha}(t ; x)\right)\right|^{2}\right)^{1 / 2},
$$

and, thanks to (4.10), this implies (4.9) for $j=1$. 
Thus, since from the inductive hypothesis and the Lipschitz continuity of $K$ the sequence $\left\{K\left(D\left[\Gamma_{\alpha}^{k}(\lambda, g)(0)\right]\right)\right\}$ and $K\left(D\left[\Gamma^{k}(\lambda, g)(0)\right]\right)$ fulfill (4.8), we can conclude that for any $R>0$

$$
\left.\lim _{\alpha \rightarrow 0} \sup _{|x|_{E} \leq R} \mid D^{j}\left(R\left(\lambda, L_{\alpha}\right)\left[K\left(D\left[\Gamma_{\alpha}^{k+1}(\lambda, g)(0)\right]\right)\right)-K\left(D\left[\Gamma_{\alpha}^{k+1}(\lambda, g)(0)\right]\right)\right]\right)(x) \mid=0 .
$$

Now, if $f \in C_{b}^{1}(H)$, for any $x \in H$ we have

$$
\begin{aligned}
& D^{j}\left[\left(R\left(\lambda, L_{\alpha}\right)-R(\lambda, L)\right)(g-K(D f))\right](x) \\
& =\int_{0}^{+\infty} e^{-\lambda t} D^{j}\left[\left(P_{t}^{\alpha}-P_{t}\right)(g-K(D f))\right](x) d t .
\end{aligned}
$$

Then, by using the estimates (3.17) and (3.18) and the limits (3.13) and (3.19), we get that

$$
\lim _{\alpha \rightarrow 0} \sup _{|x|_{E} \leq R}\left|D^{j}\left[\left(R\left(\lambda, L_{\alpha}\right)-R(\lambda, L)\right)(g-K(D f))\right](x)\right|_{H}=0
$$

for any $R>0$. As $\Gamma^{k}(\lambda, L)(0) \in C_{b}^{1}(H)$, this implies that

$$
\lim _{\alpha \rightarrow 0} \sup _{|x|_{E} \leq R}\left|D^{j}\left[\left(R\left(\lambda, L_{\alpha}\right)-R(\lambda, L)\right)\left(g-\Gamma^{k}(\lambda, g)(0)\right)\right](x)\right|_{H}=0,
$$

and recalling (4.11) we can conclude that

$$
\lim _{\alpha \rightarrow 0} \sup _{|x|_{E} \leq R}\left|D^{j}\left(\Gamma_{\alpha}^{k+1}(\lambda, g)(0)-\Gamma^{k+1}(\lambda, g)(0)\right)(x)\right|=0 .
$$

By induction this yields (4.7).

In the next proposition we show that the solution $\varphi(\lambda, g)$ of the problem (4.1) can be approximated by the solutions $\varphi_{\alpha}(\lambda, g)$ of the problems (4.6).

Proposition 4.6. Assume Hypotheses 1, 2, and 3. Then, if $\lambda_{0}$ is the constant introduced in the Proposition 4.3, for any $\lambda>\lambda_{0}$ and $g \in C_{b}(H)$ it holds that

$$
\lim _{\alpha \rightarrow 0} \sup _{|x|_{E} \leq R}\left|D^{j}\left(\varphi(\lambda, g)-\varphi_{\alpha}(\lambda, g)\right)(x)\right|_{\mathcal{L}^{j}(H)}=0, \quad j=0,1,
$$

for any $R>0$. In particular, for any $\lambda>0$ we have

$$
\lim _{\alpha \rightarrow 0} \sup _{|x|_{E} \leq R}\left|D^{j}\left[\varphi(\lambda, g)-\varphi_{\alpha}\left(\lambda+\lambda_{0}, g+\lambda_{0} \varphi(\lambda, g)\right)\right](x)\right|_{\mathcal{L}^{j}(H)}=0, \quad j=0,1 .
$$

Proof. Let us fix $\lambda_{0}$ as in Proposition 4.3. We have seen that $\varphi=\varphi(\lambda, g)$ and $\varphi_{\alpha}=\varphi_{\alpha}(\lambda, g)$ are, respectively, the unique fixed points of the mappings $\Gamma(\lambda, g)$ and $\Gamma_{\alpha}(\lambda, g)$. Since for any $\lambda>\lambda_{0}$ and $g \in C_{b}(H)$ the contraction constants of $\Gamma_{\alpha}(\lambda, g)$ are the same for all $\alpha>0$, for any $\epsilon>0$ there exists $k_{\epsilon} \in \mathbb{N}$ such that

$$
\left\|\Gamma^{k_{\epsilon}}(\lambda, g)(0)-\varphi\right\|_{1}+\sup _{\alpha>0}\left\|\Gamma_{\alpha}^{k_{\epsilon}}(\lambda, g)(0)-\varphi_{\alpha}\right\|_{1} \leq \epsilon .
$$

Thus for $j=0,1$ and $x \in H$ we have

$$
\left|D^{j}\left(\varphi-\varphi_{\alpha}\right)(x)\right| \leq \epsilon+\left|D^{j}\left(\Gamma^{k_{\epsilon}}(\lambda, g)(0)-\Gamma_{\alpha}^{k_{\epsilon}}(\lambda, g)(0)\right)(x)\right|,
$$


and due to (4.7) this implies (4.12). Now, since $\varphi(\lambda, g)=\varphi\left(\lambda+\lambda_{0}, g+\lambda_{0} \varphi(\lambda, g)\right)$, by using (4.12) we can conclude that (4.13) holds true.

Remark 4.7. For any $\alpha>0$ and $n \in \mathbb{N}$, consider the problem

$$
\lambda \varphi-L_{\alpha, n} \varphi+K_{n}(D \varphi)=g_{n},
$$

where $K_{n}(x)=K\left(P_{n} x\right)$ and $g_{n}(x)=g\left(P_{n} x\right)$ for each $n \in \mathbb{N}$ and $x \in H$. By proceeding as for the problems (4.1) and (4.6), it is possible to show that there exists $\lambda_{0}$ large enough such that for any $g \in C_{b}(H)$ and $\lambda>\lambda_{0}$ there exists a unique solution $\varphi_{\alpha, n}(\lambda, g) \in C_{b}^{1}(H)$. Such a solution is given by the unique fixed point of the mapping

$$
\Gamma_{\alpha, n}(\lambda, g)(\varphi)=R\left(\lambda, L_{\alpha, n}\right)\left(g_{n}-K_{n}(D \varphi)\right) .
$$

By using arguments analogous to those used in the Lemma 4.5, due to the estimates (3.11) and (3.18), and due to the limits (3.14) and (3.20), there exists $\lambda_{0}>0$ such that for $\lambda>\lambda_{0}$ and $g \in C_{b}(H)$ it holds that

$$
\lim _{n \rightarrow+\infty} \sup _{|x|_{H} \leq R}\left|D^{j}\left(\Gamma_{\alpha, n}^{k}(\lambda, g)(0)-\Gamma_{\alpha}^{k}(\lambda, g)(0)\right)(x)\right|_{\mathcal{L}^{j}(H)}=0, \quad j=0,1,
$$

for any $\alpha>0, k \in \mathbb{N}$, and $R>0$. Thus, by proceeding as in the proof of Proposition 4.6, due to (3.14) and (3.20) it is possible to verify that there exists $\lambda_{0}>0$ such that if $\lambda>\lambda_{0}$, then for any $\alpha>0$, and $R>0$ it holds that

$$
\lim _{n \rightarrow+\infty} \sup _{|x|_{H} \leq R}\left|D^{j}\left[\varphi_{\alpha}(\lambda, g)-\varphi_{\alpha, n}(\lambda, g)\right](x)\right|_{\mathcal{L}^{j}(H)}=0 .
$$

In the next proposition we show that if the datum $g$ belongs to $C_{b}^{1}(H)$, then the approximating problems (4.6) and (4.14) have a solution of class $C^{2}$.

Lemma 4.8. Under Hypotheses 1, 2, and 3, if $g \in C_{b}^{1}(H)$ and $\lambda>0$, then the solutions $\varphi_{\alpha}(\lambda, g)$ and $\varphi_{\alpha, n}(\lambda, g)$ of the problems (4.6) and (4.14) belong to $C_{b}^{2}(H)$. Moreover, for any $R>0$ and $\lambda>0$

$$
\sup _{\|g\|_{1} \leq R}\left\|\varphi_{\alpha}(\lambda, g)\right\|_{2}<\infty
$$

Proof. We prove the lemma only for the problem (4.6), as the proof for the problem (4.14) is identical.

As shown in Remark 4.2, $D\left(L_{\alpha}\right) \subset C_{b}^{1+\theta}(H)$ for any $\theta<(1-\epsilon) /(1+\epsilon)$. Thus, if $\varphi_{\alpha}(\lambda, g)$ is the solution of the problem (4.6), we have that $\varphi_{\alpha}(\lambda, g) \in C_{b}^{1+\theta_{0}}(H)$ for some $0<\theta_{0}<(1-\epsilon) /(1+\epsilon)$. As we have

$$
\varphi_{\alpha}(\lambda, g)=R\left(\lambda, L_{\alpha}\right)\left(g-K\left(D \varphi_{\alpha}(\lambda, g)\right)\right)
$$

by using again Remark 4.2 it follows that $\varphi_{\alpha}(\lambda, g) \in C_{b}^{1+2 \theta_{0}}(H)$. Therefore, by repeating this argument a finite number of steps we get that $\varphi_{\alpha}(\lambda, g) \in C_{b}^{2}(H)$.

The estimate (4.16) follows as above by applying (4.5) a finite number of times.

Due to (3.15), the previous lemma implies that if $g \in C_{b}^{1}(H)$, then $\varphi_{\alpha, n}=$ $\varphi_{\alpha, n}(\lambda, g)$ is a strict solution of the problem (4.14); that is,

$$
\lambda \varphi_{\alpha, n}-\mathcal{L}_{\alpha, n} \varphi_{\alpha, n}+K_{n}(D \varphi)=g_{n}
$$


where $\mathcal{L}_{\alpha, n}$ is the differential operator introduced in (3.16).

Now, for any $\varphi \in D(L)$ we define

$$
N(\varphi)=L \varphi-K(D \varphi) .
$$

In the same way, for any $\alpha>0$ and $n \in \mathbb{N}$ we define $N_{\alpha}(\varphi)=L_{\alpha} \varphi-K(D \varphi)$ and $N_{\alpha, n}(\varphi)=L_{\alpha, n} \varphi-K_{n}(D \varphi)$.

THEOREM 4.9. Under Hypotheses 1, 2, and 3, the operator $N$ defined by (4.17) is m-dissipative. Thus for any $\lambda>0$ and for any $g \in C_{b}(H)$ there exists a unique solution $\varphi(\lambda, g) \in D(L)$ for the problem (4.1).

Thanks to Proposition 4.3, in order to show that $N$ is m-dissipative, it suffices to show that $N$ is dissipative. To this purpose, we first give the following preliminary result.

Lemma 4.10. Assume that Hypotheses 1, 2, and 3 hold. Then there exists $\lambda_{0}>0$ such that for any $\lambda>\lambda_{0}$ and $\varphi_{1}, \varphi_{2} \in D\left(L_{\alpha}\right)$

$$
\left\|\varphi_{1}-\varphi_{2}\right\|_{0} \leq \frac{1}{\lambda}\left\|\lambda\left(\varphi_{1}-\varphi_{2}\right)-\left(N_{\alpha}\left(\varphi_{1}\right)-N_{\alpha}\left(\varphi_{2}\right)\right)\right\|_{0} .
$$

Proof. We set $g_{1}=\lambda \varphi_{1}-N_{\alpha}\left(\varphi_{1}\right)$ and $g_{2}=\lambda \varphi_{2}-N_{\alpha}\left(\varphi_{2}\right)$, and for any $n \in \mathbb{N}$ we set $g_{1, n}(x)=g_{1}\left(P_{n} x\right)$ and $g_{2, n}(x)=g_{2}\left(P_{n} x\right), x \in H$. Then for $\lambda$ large enough there exist $\varphi_{1, n}$ and $\varphi_{2, n}$ in $D\left(L_{\alpha, n}\right)$ such that

$$
\lambda \varphi_{1, n}-N_{\alpha, n}\left(\varphi_{1, n}\right)=g_{1, n}, \quad \lambda \varphi_{2, n}-N_{\alpha, n}\left(\varphi_{2, n}\right)=g_{2, n} .
$$

If we show that

$$
\left\|\varphi_{1, n}-\varphi_{2, n}\right\|_{0} \leq \frac{1}{\lambda}\left\|g_{1, n}-g_{2, n}\right\|_{0},
$$

we are done. Actually, for any $x \in H$ this implies that

$$
\left|\varphi_{1, n}(x)-\varphi_{2, n}(x)\right| \leq \frac{1}{\lambda}\left\|g_{1, n}-g_{2, n}\right\|_{0} \leq \frac{1}{\lambda}\left\|g_{1}-g_{2}\right\|_{0},
$$

and due to (4.15) we can take the limit as $n \rightarrow+\infty$, and we get

$$
\left|\varphi_{1}(x)-\varphi_{2}(x)\right| \leq \frac{1}{\lambda}\left\|g_{1}-g_{2}\right\|_{0} .
$$

By taking the supremum for $x \in H$, we can conclude.

Thus in order to conclude the proof we have to show that the operator $N_{\alpha, n}$ fulfills (4.18). The operator $L_{\alpha, n}$ satisfies the same conditions of the operator $\mathcal{L}$ studied in [5]; thus, thanks to [5, Proposition 7.5],

$$
\begin{aligned}
& D\left(L_{\alpha, n}\right)=\left\{\varphi \in \bigcap_{p \geq 1} W_{\text {loc }}^{2, p}\left(\mathbb{R}^{n}\right) \cap C_{b}\left(\mathbb{R}^{n}\right) ; \mathcal{L}_{\alpha, n} \varphi \in C_{b}\left(\mathbb{R}^{n}\right)\right\}, \\
& L_{\alpha, n} \varphi=\mathcal{L}_{\alpha, n} \varphi .
\end{aligned}
$$

Now we remark that

$$
\begin{aligned}
& K_{n}\left(D \varphi_{1, n}(x)\right)-K_{n}\left(D \varphi_{2, n}(x)\right) \\
& =\left\langle\int_{0}^{1} D K_{n}\left(\lambda D \varphi_{1, n}(x)+(1-\lambda) D \varphi_{2, n}(x)\right) d \lambda, D \varphi_{1, n}(x)-D \varphi_{2, n}(x)\right\rangle
\end{aligned}
$$


thus, if we set

$$
U_{\alpha, n}(x)=\int_{0}^{1} D K_{n}\left(\lambda D \varphi_{1, n}(x)+(1-\lambda) D \varphi_{2, n}(x)\right) d \lambda,
$$

we have

$$
\begin{aligned}
& \lambda\left(\varphi_{1, n}-\varphi_{2, n}\right)(x)-\mathcal{L}_{\alpha, n}\left(\varphi_{1, n}-\varphi_{2, n}\right)(x) \\
& +\left\langle U_{\alpha, n}(x), D\left(\varphi_{1, n}-\varphi_{2, n}\right)(x)\right\rangle=g_{1, n}(x)-g_{2, n}(x) .
\end{aligned}
$$

Since the function $U_{\alpha, n}$ is uniformly continuous, as $\varphi_{1, n}$ and $\varphi_{2, n}$ belong to $C_{b}^{1}(H)$, the operator $\mathcal{N}_{\alpha, n}$ defined by

$$
\mathcal{N}_{\alpha, n} \psi(x)=\mathcal{L}_{\alpha, n} \psi(x)-\left\langle U_{\alpha, n}(x), D \psi(x)\right\rangle
$$

is of the same type as the operator $\mathcal{L}$ studied in [5]. Therefore, we can adapt the proof of [5, Lemma 7.4] to the present situation, and we obtain

$$
\left\|\varphi_{1, n}-\varphi_{2, n}\right\|_{0} \leq \frac{1}{\lambda}\left\|\lambda\left(\varphi_{1, n}-\varphi_{2, n}\right)-\mathcal{N}_{\alpha, n}\left(\varphi_{1, n}-\varphi_{2, n}\right)\right\|_{0}=\frac{1}{\lambda}\left\|g_{1, n}-g_{2, n}\right\|_{0} .
$$

Proof of Theorem 4.9. Let us fix $\lambda>0$ and $\varphi_{1}, \varphi_{2} \in D(L)$, and let us define $g_{1}=\lambda \varphi_{1}-N\left(\varphi_{1}\right)$ and $g_{2}=\lambda \varphi_{2}-N\left(\varphi_{2}\right)$. If $\lambda_{0}$ is the maximum between the constant introduced in Remark 4.4 and the constant introduced in Lemma 4.10, for any $\alpha>0$ there exist $\varphi_{1, \alpha}, \varphi_{2, \alpha} \in D\left(L_{\alpha}\right)$ such that

$$
\left(\lambda+\lambda_{0}\right) \varphi_{1, \alpha}-N_{\alpha} \varphi_{1, \alpha}=g_{1}+\lambda_{0} \varphi_{1}, \quad\left(\lambda+\lambda_{0}\right) \varphi_{2, \alpha}-N_{\alpha} \varphi_{2, \alpha}=g_{2}+\lambda_{0} \varphi_{2},
$$

and

$$
\left\|\varphi_{1, \alpha}-\varphi_{2, \alpha}\right\|_{0} \leq \frac{1}{\lambda+\lambda_{0}}\left\|\left(g_{1}-g_{2}\right)+\lambda_{0}\left(\varphi_{1}-\varphi_{2}\right)\right\|_{0}
$$

Thus for any $x \in H$ we have

$$
\left|\varphi_{1, \alpha}(x)-\varphi_{2, \alpha}(x)\right| \leq \frac{1}{\lambda+\lambda_{0}}\left\|g_{1}-g_{2}\right\|_{0}+\frac{\lambda_{0}}{\lambda+\lambda_{0}}\left\|\varphi_{1}-\varphi_{2}\right\|_{0} .
$$

Now, if $x \in E$, due to (4.13) we can take the limit in the left-hand side as $\alpha$ goes to zero, and we get

$$
\left|\varphi_{1}(x)-\varphi_{2}(x)\right| \leq \frac{1}{\lambda+\lambda_{0}}\left\|g_{1}-g_{2}\right\|_{0}+\frac{\lambda_{0}}{\lambda+\lambda_{0}}\left\|\varphi_{1}-\varphi_{2}\right\|_{0} .
$$

As $\varphi_{1}$ and $\varphi_{2}$ are continuous in $H$, the estimate above holds also for $x \in H$, and by taking the supremum for $x \in H$ it follows that

$$
\left\|\varphi_{1}-\varphi_{2}\right\|_{0}-\frac{\lambda_{0}}{\lambda+\lambda_{0}}\left\|\varphi_{1}-\varphi_{2}\right\|_{0} \leq \frac{1}{\lambda+\lambda_{0}}\left\|g_{1}-g_{2}\right\|_{0}
$$

so that

$$
\left\|\varphi_{1}-\varphi_{2}\right\|_{0} \leq \frac{1}{\lambda}\left\|g_{1}-g_{2}\right\|_{0}
$$


4.2. Locally Lipschitz hamiltonian $\boldsymbol{K}$. We first prove an a priori estimate which is crucial in order to prove the $m$-dissipativity of the operator $N$ in the case of a locally Lipschitz hamiltonian $K$.

Proposition 4.11. Assume that Hypotheses 1, 2, and 3 hold. Then there exists some $\mu_{0}>0$, which does not depend on $K$, such that if $g \in C_{b}^{1}(H)$ and $\lambda>\mu_{0}$, then

$$
\|D \varphi(\lambda, g)\|_{0} \leq\|D g\|_{0} .
$$

Proof. Let us fix $\lambda, \mu>0$ and $g \in C_{b}^{1}(H)$, and let us consider $\varphi_{\alpha}=\varphi_{\alpha}(\lambda+\mu, g+$ $\mu \varphi(\lambda, g))$ and $\varphi_{\alpha, n}=\varphi_{\alpha, n}(\lambda+\mu, g+\mu \varphi(\lambda, g))$. Since $g \in C_{b}^{1}(H)$, then $\varphi_{\alpha, n}$ belongs to $C_{b}^{2}(H)$, and it is a strict solution of the problem

$$
(\lambda+\mu) \varphi-N_{\alpha, n}(\varphi)=g_{n}+\mu \varphi_{n}(\lambda, g)
$$

where $\varphi_{n}(\lambda, g)(x)=\varphi(\lambda, g)\left(P_{n} x\right)$. The problem above can be rewritten as

$$
\begin{aligned}
& (\lambda+\mu) \varphi(x)-\frac{1}{2} \sum_{h=1}^{n} \lambda_{h}^{2} D_{h}^{2} \varphi(x)-\sum_{h, k=1}^{n} a_{h k} x_{k} D_{h} \varphi(x) \\
& -\left\langle F_{\alpha}\left(P_{n} x\right), D \varphi(x)\right\rangle_{H}+K\left(P_{n} D \varphi(x)\right)=g\left(P_{n} x\right)+\mu \varphi(\lambda, g)\left(P_{n} x\right),
\end{aligned}
$$

where $D_{h} \varphi(x)=\left\langle D \varphi(x), e_{h}\right\rangle_{H}$ and $a_{h k}=\left\langle A e_{k}, e_{h}\right\rangle_{H}$. By differentiating with respect to $x_{j}$, by setting $\psi_{h}=D_{h} \varphi$, for $h=1, \ldots, n$, and by multiplying each side by $\psi_{j}$, we get

$$
\begin{aligned}
& (\lambda+\mu) \psi_{j}^{2}-\frac{1}{2} \sum_{h=1}^{n} \lambda_{h}^{2} \psi_{j} D_{h}^{2} \psi_{j}-\sum_{h, k=1}^{n} a_{h k} x_{k} \psi_{j} D_{h} \psi_{j}-\sum_{h=1}^{n} a_{h j} \psi_{h} \psi_{j} \\
& -\sum_{h=1}^{n}\left\langle F_{\alpha, n}, e_{h}\right\rangle\left\langle\psi_{j} D \psi_{j}, e_{h}\right\rangle-\sum_{h=1}^{n}\left\langle D F_{\alpha, n} e_{j} \psi_{j}, e_{h}\right\rangle \psi_{h} \\
& +\sum_{h=1}^{n} D_{h} K\left(P_{n} D \varphi_{\alpha, n}\right) \psi_{j} D_{h} \psi_{j}=\left\langle D g_{n}, e_{j} \psi_{j}\right\rangle+\mu\left\langle D \varphi_{n}(\lambda, g), e_{j} \psi_{j}\right\rangle .
\end{aligned}
$$

Then we sum up over $j$ and by setting $z(x)=\left|D \varphi_{\alpha, n}(x)\right|_{H}^{2}$ and by taking into account that

$$
\left(D_{h}^{2} \psi_{j}\right) \psi_{j}=\frac{1}{2} D_{h}^{2}\left(\psi_{j}^{2}\right)-\left(D_{h} \psi_{j}\right)^{2}
$$

we have

$$
\begin{aligned}
& 2(\lambda+\mu) z(x)-\frac{1}{2} \operatorname{Tr}\left[Q_{n}^{2} D^{2} z(x)\right]+\sum_{h, j=1}^{n} \lambda_{h}^{2}\left(D_{h} \psi_{j}\right)^{2}(x)-\left\langle A_{n} x, D z(x)\right\rangle \\
& -2\left\langle A_{n} D \varphi_{\alpha, n}(x), D \varphi_{\alpha, n}(x)\right\rangle+\left\langle D K\left(D \varphi_{\alpha, n}(x)\right), D z(x)\right\rangle-\left\langle F_{\alpha}\left(P_{n} x\right), D z(x)\right\rangle \\
& -2\left\langle D F_{\alpha}\left(P_{n} x\right) D \varphi_{\alpha, n}(x), D \varphi_{\alpha, n}(x)\right\rangle=2\left\langle D g\left(P_{n} x\right)+\mu D \varphi(\lambda, g)\left(P_{n} x\right), D \varphi_{\alpha, n}(x)\right\rangle .
\end{aligned}
$$


Therefore, by using (2.3) and (2.4) it follows that

$$
\begin{aligned}
& 2(\lambda+\mu) z(x)-\frac{1}{2} \operatorname{Tr}\left[Q_{n}^{2} D^{2} z(x)\right]-\left\langle A_{n} x, D z(x)\right\rangle-\left\langle F_{\alpha}\left(P_{n} x\right), D z(x)\right\rangle \\
& +\left\langle D K\left(D \varphi_{\alpha, n}(x)\right), D z(x)\right\rangle \leq 2\left\langle D g(x)+\mu D \varphi(\lambda, g)\left(P_{n} x\right), D \varphi_{\alpha, n}\left(P_{n} x\right)\right\rangle+\gamma z(x) \\
& \leq 2\left(\|D g\|_{0}+\mu\|D \varphi(\lambda, g)\|_{0}\right)\left|D \varphi_{\alpha, n}(x)\right|_{H}+\gamma z(x)
\end{aligned}
$$

for a suitable constant $\gamma \in \mathbb{R}$ depending only on $F$ and $A$.

Now let us consider the equation

$$
d y(t)=\left[A_{n} y(t)+F_{\alpha, n}(y(t))+U_{\alpha, n}(y(t))\right] d t+Q_{n} d w(t), \quad y(0)=P_{n} x,
$$

where $U_{\alpha, n}(x)=-D K\left(D \varphi_{\alpha, n}(x)\right)$ for any $x \in H$. If $g \in C_{b}^{1}(H)$, then $\varphi_{\alpha, n} \in C_{b}^{2}(H)$, and then the mapping $U_{\alpha, n}: H \rightarrow H$ is Lipschitz continuous. This implies that there exists a unique strong solution $y_{\alpha, n}(\cdot ; x) \in L^{2}(\Omega ; C([0,+\infty) ; H))$ for (4.20). If we denote by $R_{t}^{\alpha, n}$ the corresponding transition semigroup, it is possible to show that the solution of the problem

$$
\begin{aligned}
& (2(\lambda+\mu)-\gamma) \psi(x)-\frac{1}{2} \operatorname{Tr}\left[Q_{n}^{2} D^{2} \psi(x)\right]-\left\langle A_{n} x, D \psi(x)\right\rangle-\left\langle F_{\alpha}\left(P_{n} x\right), D \psi(x)\right\rangle \\
& +\left\langle D K\left(D \varphi_{\alpha, n}(x)\right), D \psi(x)\right\rangle=2\left(\|D g\|_{0}+\mu\|D \varphi(\lambda, g)\|_{0}\right)\left|D \varphi_{\alpha, n}(x)\right|_{H}
\end{aligned}
$$

for any $\lambda>\gamma$ is given by

$$
\psi(x)=2\left(\|D g\|_{0}+\mu\|D \varphi(\lambda, g)\|_{0}\right) \int_{0}^{+\infty} e^{-(2(\lambda+\mu)-\gamma) t} R_{t}^{\alpha, n}\left(\left|D \varphi_{\alpha, n}\right|_{H}\right)(x) d t .
$$

(See [5] for a proof.) Thus by a comparison argument we have that

$$
\left|D \varphi_{\alpha, n}(x)\right|_{H}^{2} \leq \frac{2}{2(\lambda+\mu)-\gamma}\left(\|D g\|_{0}+\mu\|D \varphi(\lambda, g)\|_{0}\right)\left|D \varphi_{\alpha, n}(x)\right|_{H},
$$

and if we take $\lambda>1+\gamma / 2=\mu_{0}$, it follows that

$$
\left|D \varphi_{\alpha, n}(\lambda+\mu, g+\mu \varphi(\lambda, g))(x)\right|_{H} \leq \frac{1}{1+\mu}\left(\|D g\|_{0}+\mu\|D \varphi(\lambda, g)\|_{0}\right) .
$$

Due to (4.13) and (4.15), if $\mu$ is large enough, we can take first the limit as $n$ goes to infinity and then the limit as $\alpha$ goes to zero, and for any $x \in E$ we get

$$
|D \varphi(x)|_{H} \leq \frac{1}{1+\mu}\left(\|D g\|_{0}+\mu\|D \varphi(\lambda, g)\|_{0}\right) .
$$

As $\varphi(\lambda, g) \in C_{b}^{1}(H)$, the same estimate holds for $x \in H$, and then, by taking the supremum for $x \in H$, we get

$$
\|D \varphi(\lambda, g)\|_{0} \leq \frac{1}{1+\mu}\left(\|D g\|_{0}+\mu\|D \varphi(\lambda, g)\|_{0}\right)
$$

which immediately yields (4.19). 
Remark 4.12. It is immediate to check that the proof of the previous proposition adapts to the problem (4.6). Thus there exists $\lambda_{0}>0$, which is clearly independent of $\alpha>0$, such that for any $\lambda>\lambda_{0}$ and $g \in C_{b}^{1}(H)$

$$
\left\|D \varphi_{\alpha}(\lambda, g)\right\|_{0} \leq\|D g\|_{0}
$$

From now on we shall assume that $K$ fulfills the following assumption.

Hypothesis 4. The hamiltonian $K: H \rightarrow \mathbb{R}$ is Fréchet differentiable and is locally Lipschitz continuous, together with its derivative. Moreover, $K(0)=0$.

We want to show that under the hypotheses above the problem (4.1) admits a unique solution for any $\lambda>\mu_{0}$ and $g \in C_{b}^{1}(H)$. To this purpose, for any $r>0$ let $K_{r}$ be a Fréchet differentiable function such that

$$
K_{r}(x)= \begin{cases}K(x) & \text { if }|x|_{H} \leq r \\ K\left(\frac{(r+1) x}{|x|_{H}}\right) & \text { if }|x|_{H}>r+1 .\end{cases}
$$

It is immediate to check that $K_{r}$ is Lipschitz continuous, together with its derivative, for each $r>0$, and $K_{r}(x)=K(x)$ if $|x|_{H} \leq r$.

THEOREM 4.13. Under Hypotheses 1, 2, and 4 there exists $\mu_{0}>0$ such that for any $\lambda>\mu_{0}$ and $g \in C_{b}^{1}(H)$ there exists a unique solution $\varphi(\lambda, g) \in D(L)$ for the problem (4.1).

Proof. For any $r>0$ and $g \in C_{b}^{1}(H)$ we define $\varphi_{r}(\lambda, g)$ as the solution of the problem $\lambda \varphi-L \varphi+K_{r}(D \varphi)=g$. Due to Proposition 4.11 there exists $\mu_{0}>0$ such that for any $\lambda>\mu_{0}$

$$
\sup _{r>0}\left\|D \varphi_{r}(\lambda, g)\right\|_{0} \leq\|D g\|_{0}
$$

Thus, if we fix $r>\|g\|_{1}$, we have that $K_{r}\left(D \varphi_{r}(\lambda, g)\right)=K\left(D \varphi_{r}(\lambda, g)\right)$, and then

$$
\lambda \varphi_{r}(\lambda, g)-L \varphi_{r}(\lambda, g)+K\left(D \varphi_{r}(\lambda, g)\right)=g .
$$

Remark 4.14. The operator $N$ is dissipative. Actually, fix $\lambda>0$ and $\varphi_{1}, \varphi_{2} \in$ $D(L)$, and define $g_{i}=\lambda \varphi_{i}-N\left(\varphi_{i}\right)$ for $i=1,2$. If we take $r \geq \max \left(\left\|\varphi_{1}\right\|_{1},\left\|\varphi_{2}\right\|_{1}\right)$, we have

$$
g_{i}=\lambda \varphi_{i}-L \varphi_{i}+K_{r}\left(D \varphi_{i}\right), \quad i=1,2 .
$$

Thus we can apply Theorem 4.9 to the hamiltonian $K_{r}$, and we get

$$
\left\|\varphi_{1}-\varphi_{2}\right\|_{0} \leq \frac{1}{\lambda}\left\|g_{1}-g_{2}\right\|_{0}
$$

so that $N$ is dissipative. In particular, $N$ is closable, and its closure $\bar{N}$ is $m$-dissipative, so that for any $\lambda>0$ and $g \in C_{b}(H)$ there exists a unique solution to the problem

$$
\lambda \varphi-\bar{N}(\varphi)=g
$$


5. Application to the control problem. Let $k: H \rightarrow(-\infty,+\infty]$ be a measurable mapping such that its Legendre transform

$$
K(x)=\sup \left\{-\langle x, y\rangle_{H}-k(y) ; y \in H\right\}, \quad x \in H,
$$

fulfills Hypothesis 4. It is possible to show that if $k$ is strictly convex and continuously Fréchet differentiable, if

$$
\lim _{|y|_{H \rightarrow+\infty}} \frac{k(y)}{|y|_{H}}=0
$$

and if $D k: H \rightarrow \mathcal{L}(H)$ has a continuous inverse which is Lipschitz continuous on bounded subsets of $H$, then Hypothesis 4 is verified. An easy example is given by $k(y)=|y|_{H}^{2}$.

For any $\lambda>0$ and $g \in C_{b}(H)$ we consider the cost functional

$$
J(x ; z)=\mathbb{E} \int_{0}^{+\infty} e^{-\lambda t}[g(y(t))+k(z(t))] d t,
$$

where $y(t)=y(t ; x, z)$ is the unique solution of the system (3.1). The corresponding value function is defined as

$$
V(x)=\inf \left\{J(x ; z) ; z \in L^{2}\left(\Omega ; L^{2}(0,+\infty ; H)\right) \text { adapted }\right\} .
$$

Our aim is to prove that if $\varphi$ is the unique solution of the Hamilton-Jacobi equation (4.1), then $V(x)=\varphi(x)$ for any $x \in H$. To this purpose we first prove the following preliminary result.

Lemma 5.1. Assume Hypotheses 1, 2, and 4. If $\varphi=\varphi(\lambda, g)$ is the solution of the problem (4.1) in $C_{b}^{1}(H)$ and if $y(t)=y(t ; x, z)$ is the solution of the controlled system (3.1), we have

$$
J(x ; z)=\varphi(x)+\mathbb{E} \int_{0}^{+\infty} e^{-\lambda t}\left[K(D \varphi(y(t)))+\langle z(t), D \varphi(y(t))\rangle_{H}+k(z(t))\right] d t .
$$

Proof. If $r \geq\|D \varphi(\lambda, g)\|_{0}$ and if $K_{r}$ is defined as in (4.21), then we have $K(D \varphi(x))=K_{r}(D \varphi(x))$ for any $x \in H$, and the problem (4.1) can be rewritten as

$$
\lambda \varphi-L \varphi+K_{r}(D \varphi)=g .
$$

Now we fix a sequence $\left\{g_{k}\right\} \subset C_{b}^{1}(H)$ converging to $g$ in $C_{b}(H)$ and for any $k, n \in \mathbb{N}$ and $\alpha>0$ we denote by $\varphi_{\alpha, n}^{k}=\varphi_{\alpha, n}^{k}\left(\lambda+\mu, g_{k}+\mu \varphi(\lambda, g)\right)$ the solution of the problem

$$
(\lambda+\mu) \varphi-L_{\alpha, n} \varphi+K_{r, n}(D \varphi)=g_{k, n}+\mu \varphi_{n}(\lambda, g),
$$

where $K_{r, n}(x)=K_{r}\left(P_{n} x\right), g_{k, n}(x)=g_{k}\left(P_{n} x\right)$, and $\varphi_{n}(\lambda, g)(x)=\varphi(\lambda, g)\left(P_{n} x\right)$, and $\mu$ is some positive constant to be determined later. Since $g_{k}$ and $\varphi(\lambda, g)$ are continuously differentiable, due to Lemma 4.8 we have that $\varphi_{\alpha, n}^{k}$ belongs to $C_{b}^{2}(H)$. Then, since $y_{\alpha, n}(t ; x, z)$ is a strong solution of the problem (3.9), we can apply the Itô formula to the mapping $t \mapsto e^{-\lambda t} \varphi_{\alpha, n}^{k}\left(y_{\alpha, n}(t)\right)$, and we get

$$
\begin{aligned}
& d\left(e^{-\lambda t} \varphi_{\alpha, n}^{k}\left(y_{\alpha, n}(t)\right)\right)=e^{-\lambda t}\left\langle D \varphi_{\alpha, n}^{k}\left(y_{\alpha, n}(t)\right), Q_{n} d w(t)\right\rangle_{H} \\
& +e^{-\lambda t}\left(\left(\mathcal{L}_{\alpha, n}-\lambda\right) \varphi_{\alpha, n}^{k}\left(y_{\alpha, n}(t)\right)+\left\langle P_{n} z(t), D \varphi_{\alpha, n}^{k}\left(y_{\alpha, n}(t)\right)\right\rangle_{H}\right) .
\end{aligned}
$$


Recalling that $\varphi_{\alpha, n}^{k}$ is the solution of (5.3) and that (3.15) holds, we have

$$
\left(\mathcal{L}_{\alpha, n}-\lambda\right) \varphi_{\alpha, n}^{k}=\mu \varphi_{\alpha, n}^{k}+K_{r, n}\left(D \varphi_{\alpha, n}^{k}\right)-g_{k, n}-\mu \varphi_{n}(\lambda, g) .
$$

Then, by integrating with respect to $t \in[0, T]$ and by taking the expectation, we get

$$
\begin{aligned}
& e^{-\lambda T} P_{T}^{\alpha, n} \varphi_{\alpha, n}^{k}-\varphi_{\alpha, n}^{k}=\mu \mathbb{E} \int_{0}^{T} e^{-\lambda t}\left(\varphi_{\alpha, n}^{k}-\varphi(\lambda, g)\right)\left(y_{\alpha, n}(t)\right) d t \\
& +\mathbb{E} \int_{0}^{T} e^{-\lambda t}\left(K_{r}\left(D \varphi_{\alpha, n}^{k}\left(y_{\alpha, n}(t)\right)\right)-g_{k}\left(y_{\alpha, n}(t)\right)+\left\langle z(t), D \varphi_{\alpha, n}^{k}\left(y_{\alpha, n}(t)\right)\right\rangle_{H}\right) d t .
\end{aligned}
$$

Due to (3.10) and (4.15), if $\mu$ is large enough, we can take the limit as $n$ goes to infinity, and we get

$$
\begin{aligned}
& e^{-\lambda T} P_{T}^{\alpha} \varphi_{\alpha}^{k}(x)-\varphi_{\alpha}^{k}(x)=\mu \mathbb{E} \int_{0}^{T} e^{-\lambda t}\left(\varphi_{\alpha}^{k}-\varphi(\lambda, g)\right)\left(y_{\alpha}(t)\right) d t \\
& =\mathbb{E} \int_{0}^{T} e^{-\lambda t}\left[K_{r}\left(D \varphi_{\alpha}^{k}\left(y_{\alpha}(t)\right)\right)-g_{k}\left(y_{\alpha}(t)\right)+\left\langle z(t), D \varphi_{\alpha}^{k}\left(y_{\alpha}(t)\right)\right\rangle_{H}\right] d t,
\end{aligned}
$$

where $\varphi_{\alpha}^{k}=\varphi_{\alpha}^{k}\left(\lambda+\mu, g_{k}+\mu \varphi(\lambda, g)\right)$ is the solution of the problem

$$
(\lambda+\mu) \varphi-L_{\alpha} \varphi+K_{r}(D \varphi)=g_{k}+\mu \varphi(\lambda, g) .
$$

By taking the limit as $T$ goes to infinity, this yields

$$
\begin{aligned}
& -\varphi_{\alpha}^{k}=\mu \mathbb{E} \int_{0}^{+\infty} e^{-\lambda t}\left(\varphi_{\alpha}^{k}-\varphi(\lambda, g)\right)\left(y_{\alpha}(t)\right) d t \\
& +\mathbb{E} \int_{0}^{+\infty} e^{-\lambda t}\left[K_{r}\left(D \varphi_{\alpha}^{k}\left(y_{\alpha}(t)\right)\right)-g_{k}\left(y_{\alpha}(t)\right)+\left\langle z(t), D \varphi_{\alpha}^{k}\left(y_{\alpha}(t)\right)\right\rangle_{H}\right] d t .
\end{aligned}
$$

We remark that for any $h, k \in \mathbb{N}$ and $\alpha>0$ we have

$$
\varphi_{\alpha}^{k}-\varphi_{\alpha}^{h}=R\left(\lambda+\mu, L_{\alpha}\right)\left[g_{k}-g_{h}-\left(K_{r}\left(D \varphi_{\alpha}^{k}\right)-K_{r}\left(D \varphi_{\alpha}^{h}\right)\right)\right],
$$

and then, due to (4.2),

$$
\left\|\varphi_{\alpha}^{k}-\varphi_{\alpha}^{h}\right\|_{1} \leq \rho(\lambda+\mu)\left(\left\|g_{k}-g_{h}\right\|_{0}+c_{r}\left\|D \varphi_{\alpha}^{k}-D \varphi_{\alpha}^{h}\right\|_{0}\right),
$$

where $c_{r}$ is the Lipschitz constant of $K_{r}$. Therefore, if $\mu$ is sufficiently large, we have $\rho(\lambda+\mu) c_{r}<1$, so that

$$
\left\|\varphi_{\alpha}^{k}-\varphi_{\alpha}^{h}\right\|_{1} \leq \frac{\rho(\lambda+\mu)}{1-\rho(\lambda+\mu) c_{r}}\left\|g_{k}-g_{h}\right\|_{0}
$$

This means that the sequence $\left\{\varphi_{\alpha}^{k}\right\}$ converges to some $\varphi_{\alpha}$ in $C_{b}^{1}(H)$. It is immediate to check that $\varphi_{\alpha}$ coincides with $\varphi_{\alpha}(\lambda+\mu, g+\mu \varphi(\lambda, g))$, and then, by taking the limit as $k$ goes to infinity in (5.4), due to the dominated convergence theorem we can conclude that

$$
\begin{aligned}
& -\varphi_{\alpha}=\mu \mathbb{E} \int_{0}^{+\infty} e^{-\lambda t}\left(\varphi_{\alpha}-\varphi(\lambda, g)\right)\left(y_{\alpha}(t)\right) d t \\
& +\mathbb{E} \int_{0}^{+\infty} e^{-\lambda t}\left[K_{r}\left(D \varphi_{\alpha}\left(y_{\alpha}(t)\right)\right)-g\left(y_{\alpha}(t)\right)+\left\langle z(t), D \varphi_{\alpha}\left(y_{\alpha}(t)\right)\right\rangle_{H}\right] d t .
\end{aligned}
$$


If $x \in E$ and $z \in L^{p}\left(\Omega ; L^{\infty}(0,+\infty ; H)\right)$ with $p$ as in the Proposition 3.2, we can use (3.7) and (4.13), and by taking the limit as $\alpha$ goes to zero we have

$$
\varphi(x)+\mathbb{E} \int_{0}^{+\infty} e^{-\lambda t}\left[K(D \varphi(y(t)))-g(y(t))+\langle z(t), D \varphi(y(t))\rangle_{H}\right] d t=0 .
$$

Notice that here we have replaced $K_{r}$ by $K$, as we fixed $r \geq\|D \varphi(\lambda, g)\|_{0}$. Since $\varphi \in$ $C_{b}^{1}(H)$ and $y(t ; x, z)$ depends continuously on $x \in H$ and $z \in L^{2}\left(\Omega ; L^{2}(0,+\infty ; H)\right)$, the same identity holds for $x \in H$ and $z \in L^{2}\left(\Omega ; L^{2}(0,+\infty ; H)\right)$. Then, recalling how $J(x ; z)$ is defined, if we rearrange all terms, we get (5.2).

TheOREm 5.2. Assume that Hypotheses 1, 2, and 4 hold. Then there exists $\mu_{0}$ such that for any $\lambda>\mu_{0}$ and $g \in C_{b}^{1}(H)$ the value function $V$ corresponding to the cost functional (5.1) coincides with the solution $\varphi(\lambda, g)$ of the Hamilton-Jacobi equation (4.1).

Moreover, for any $x \in E$ we have

$$
V(x)=\lim _{\alpha \rightarrow 0} \min \left\{J_{\alpha}(x ; z) ; z \in L^{2}\left(\Omega ; L^{2}(0,+\infty ; H)\right) \text { adapted }\right\},
$$

where $\left\{J_{\alpha}(x, z)\right\}$ is a sequence of cost functionals which admit unique optimal controls and states and whose value functions $V_{\alpha}$ coincide with the solution of the problems

$$
\left(\lambda+\lambda_{0}\right) \varphi-L_{\alpha} \varphi+K_{r}(D \varphi)=g+\lambda_{0} \varphi(\lambda, g)
$$

for some $\lambda_{0}>0$ large enough and $r \geq\|D \varphi(\lambda, g)\|_{0}$.

Proof. In Theorem 4.13 we have seen that, if $\lambda>\mu_{0}$ and $g \in C_{b}^{1}(H)$, there exists a unique solution $\varphi(\lambda, g) \in C_{b}^{1}(H)$ for (4.1). Due to (5.2) and to the definition of $K$, we have that $V(x) \geq \varphi(\lambda, g)(x)$ for any $x \in H$. Now we try to prove the opposite inequality. To this purpose we proceed by approximation.

We fix $r \geq\|D \varphi(\lambda, g)\|_{0}$, and for any $\alpha>0$ we define the cost functional

$$
\begin{aligned}
& J_{\alpha}(x ; z)=\mathbb{E} \int_{0}^{+\infty} e^{-\lambda t}\left[g\left(y_{\alpha}(t ; x, z)\right)+k(z(t))\right] d t \\
& +\lambda_{0} \mathbb{E} \int_{0}^{+\infty} e^{-\lambda t}\left[\left(\varphi(\lambda, g)-\varphi_{\alpha}\right)\left(y_{\alpha}(t ; x, z)\right)\right] d t \\
& +\mathbb{E} \int_{0}^{+\infty} e^{-\lambda t}\left[K\left(D \varphi_{\alpha}\left(y_{\alpha}(t ; x, z)\right)\right)-K_{r}\left(D \varphi_{\alpha}\left(y_{\alpha}(t ; x, z)\right)\right)\right] d t,
\end{aligned}
$$

where $\varphi_{\alpha}=\varphi_{\alpha}\left(\lambda+\lambda_{0}, g+\lambda_{0} \varphi(\lambda, g)\right)$ is the solution of the problem

$$
\left(\lambda+\lambda_{0}\right) \varphi-L_{\alpha} \varphi+K_{r}(D \varphi)=g+\lambda_{0} \varphi(\lambda, g),
$$

and $\lambda_{0}$ is the constant introduced in Proposition 4.3 corresponding to the hamiltonian $K_{r}$. We denote by $V_{\alpha}(x)$ the corresponding value function. Thanks to (5.6) we easily have that $V_{\alpha}(x) \geq \varphi_{\alpha}(x)$ for any $x \in H$. In fact, it is possible to show that $V_{\alpha}(x)=\varphi_{\alpha}(x)$. Indeed, for each $x \in H$ the function

$$
H \rightarrow \mathbb{R}, \quad z \mapsto-\left\langle z, D \varphi_{\alpha}(x)\right\rangle_{H}-k(z)
$$

attains its maximum at $z=-D K\left(D \varphi_{\alpha}(x)\right)$. Then, if we show that the closed loop equation

$$
d y(t)=\left[A y(t)+F_{\alpha}(y(t))-D K\left(D \varphi_{\alpha}(y(t))\right)\right] d t+Q d w(t), \quad y(0)=x,
$$


has a unique adapted solution $y_{\alpha}^{\star}(t)$, we have that for the control

$$
z_{\alpha}^{\star}(t)=-D K\left(D \varphi_{\alpha}\left(y_{\alpha}^{\star}(t)\right)\right)
$$

it holds that $J_{\alpha}\left(x, z_{\alpha}^{\star}\right)=\varphi_{\alpha}(x)$. This means that $V_{\alpha}(x)=\varphi_{\alpha}(x)$, and there exists a unique optimal control and a unique optimal state for the minimizing problem corresponding to the cost functional $J_{\alpha}(x ; z)$.

If $g \in C_{b}^{1}(H)$, then due to Lemma $4.8 \varphi_{\alpha} \in C_{b}^{2}(H)$, so that the mapping

$$
U_{\alpha}: H \rightarrow H, \quad x \mapsto-D K\left(D \varphi_{\alpha}(x)\right)
$$

is Lipschitz continuous. This implies that the closed loop equation admits a unique solution.

For any $\alpha>0$ the optimal control relative to the functional $J_{\alpha}(x ; z)$ is $z_{\alpha}^{\star}(t)=$ $-D K\left(D \varphi_{\alpha}\left(y_{\alpha}^{\star}(t)\right)\right)$. According to Proposition 4.11 we have

$$
\left\|D \varphi_{\alpha}\right\|_{0} \leq\|D g\|_{0}+\lambda_{0}\|D \varphi(\lambda, g)\|_{0},
$$

and then, since $D K$ is bounded on bounded sets, there exists $R>0$ such that

$$
\sup _{\alpha>0} \sup _{t \geq 0}\left|z_{\alpha}^{\star}(t)\right|_{H}=R, \quad \mathbb{P} \text {-a.s. }
$$

This implies that

$$
V_{\alpha}(x)=\inf \left\{J_{\alpha}(x ; z): z \in \mathcal{M}_{R}^{2}\right\},
$$

where $\mathcal{M}_{R}^{2}$ is the subset of admissible controls introduced in (3.8).

Now, recalling Proposition 4.6, we have that for any $x \in E$

$$
\lim _{\alpha \rightarrow 0} V_{\alpha}(x)=\lim _{\alpha \rightarrow 0} \varphi_{\alpha}\left(\lambda+\lambda_{0}, g+\lambda_{0} \varphi(\lambda, g)\right)(x)=\varphi(\lambda, g)(x) .
$$

Thus, if we show that

$$
\lim _{\alpha \rightarrow 0} \sup _{z \in \mathcal{M}_{R}^{2}}\left|J_{\alpha}(x ; z)-J(x ; z)\right|=0,
$$

it immediately follows that $V(x)=\varphi(\lambda, g)(x)$ for $x \in E$.

Due to Proposition 3.2, we have that

$$
\lim _{\alpha \rightarrow 0} \mathbb{E}\left|g\left(y_{\alpha}(t ; x, z)\right)-g(y(t ; x, z))\right|=0,
$$

uniformly for $(t, x)$ in bounded sets of $[0,+\infty) \times E$ and $z \in \mathcal{M}_{R}^{2}$. Hence, if we fix $\epsilon>0$ and $M>0$ such that

$$
\int_{M}^{+\infty} e^{-\lambda t} d t \leq \frac{\epsilon}{2\|g\|_{0}}
$$

we have

$$
\begin{aligned}
& \mathbb{E} \int_{0}^{+\infty} e^{-\lambda t}\left[g\left(y_{\alpha}(t ; x, z)\right)-g(y(t ; x, z))\right] d t \\
& \leq \epsilon+\int_{0}^{M} e^{-\lambda t} \mathbb{E}\left|g\left(y_{\alpha}(t ; x, z)\right)-g(y(t ; x, z))\right| d t,
\end{aligned}
$$


so that, due to the arbitrariness of $\epsilon>0$,

$$
\lim _{\alpha \rightarrow 0} \sup _{z \in \mathcal{M}_{R}^{2}} \mathbb{E} \int_{0}^{+\infty} e^{-\lambda t}\left[g\left(y_{\alpha}(t ; x, z)\right)-g(y(t ; x, z))\right] d t=0 .
$$

Thanks to Lemma 4.8, we have that for $j=0,1$

$$
\lim _{\alpha \rightarrow 0} \sup _{|x|_{E} \leq R}\left|D^{j}\left(\varphi_{\alpha}-\varphi\right)(x)\right|_{\mathcal{L}^{j}(H)}=0 .
$$

Moreover, thanks to (3.6),

$$
\sup _{z \in \mathcal{M}_{R}^{2}} \sup _{t \in[0, T]}\left|y_{\alpha}(t ; x, z)\right|_{E}<+\infty, \quad \mathbb{P} \text {-a.s. }
$$

for any $T>0$. Thus, by using the same arguments as above, we have

$$
\lim _{\alpha \rightarrow 0} \sup _{z \in \mathcal{M}_{R}^{2}} \mathbb{E} \int_{0}^{+\infty} e^{-\lambda t}\left[\left(\varphi_{\alpha}-\varphi\right)\left(y_{\alpha}(t ; x, z)\right)\right] d t=0 .
$$

Finally, since the sequence $\left\{\varphi_{\alpha}\right\}$ is bounded in $C_{b}^{1}(H)$, recalling that $K$ and $K_{r}$ are bounded on bounded sets and $K(D \varphi(x))=K_{r}(D \varphi(x))$ for any $x \in H$, by using (5.9) and by arguing as above, we have

$$
\lim _{\alpha \rightarrow 0} \sup _{z \in \mathcal{M}_{R}^{2}} \mathbb{E} \int_{0}^{+\infty} e^{-\lambda t}\left[K\left(D \varphi_{\alpha}\left(y_{\alpha}(t ; x, z)\right)\right)-K_{r}\left(D \varphi_{\alpha}\left(y_{\alpha}(t ; x, z)\right)\right)\right] d t=0 .
$$

Therefore, we can conclude that (5.8) holds for any $x \in E$, and then $V(x)=\varphi(x)$ for $x \in E$.

Now assume that $x \in H$. We fix a sequence $\left\{x_{n}\right\} \subset E$ converging to $x$ in $H$. For each $n \in \mathbb{N}$ we have $V\left(x_{n}\right)=\varphi\left(x_{n}\right)$ and

$$
J\left(x_{n} ; z\right)-J(x ; z)=\mathbb{E} \int_{0}^{+\infty} e^{-\lambda t}\left[g\left(y\left(t ; x_{n}, z\right)\right)-g(y(t ; x, z))\right] d t .
$$

Then, due to (3.4), if $\varphi \in C_{b}^{1}(H)$, we easily get

$$
\lim _{n \rightarrow+\infty} \sup _{z \in \mathcal{M}_{R}^{2}} J\left(x_{n} ; z\right)=J(x ; z),
$$

so that we can conclude that $V(x)=\varphi(x)$ for any $x \in H$.

We have seen that if we assume the hamiltonian $K$ to be Lipschitz continuous, then for any $\lambda>0$ and $g \in C_{b}(H)$ there exists a unique solution $\varphi(\lambda, g)$ in $D(L) \subset$ $C_{b}^{1}(H)$ to the problem (4.1). This allows us to have a stronger version of the previous theorem in the case of Lipschitz $K$.

TheOREm 5.3. Assume that Hypotheses 1, 2, and 3 hold. Then for any $\lambda>0$ and $g \in \operatorname{Lip}_{b}(H)$ the value function $V$ corresponding to the cost functional (5.1) coincides with the solution $\varphi(\lambda, g)$ of the Hamilton-Jacobi equation (4.1).

Moreover, for any $x \in E$ we have

$$
V(x)=\lim _{\alpha \rightarrow 0} \min \left\{J_{\alpha}(x ; z) ; z \in L^{2}\left(\Omega ; L^{2}(0,+\infty ; H)\right) \text { adapted }\right\},
$$


where $\left\{J_{\alpha}(x, z)\right\}$ is a sequence of cost functionals which admit unique optimal controls and states and whose value functions $V_{\alpha}$ coincide with the solution of the problems

$$
\left(\lambda+\lambda_{0}\right) \varphi-L_{\alpha} \varphi+K(D \varphi)=g+\lambda_{0} \varphi(\lambda, g)
$$

for some $\lambda_{0}>0$.

Proof. By arguing as in the proof of the previous theorem, we have the thesis for any $g \in C_{b}^{1}(H)$ and $\lambda>0$. Thus, in order to conclude, we have to show that for any $g \in \operatorname{Lip}_{b}(H)$ the approximating closed loop equation

$$
d u(t)=\left[A u(t)+F_{\alpha}(u(t))-D K\left(D \varphi_{\alpha}(u(t))\right)\right] d t+Q d w(t), \quad u(0)=x,
$$

admits a unique adapted solution $u_{\alpha}^{\star}(t)$.

If $g \in \operatorname{Lip}_{b}(H)$, we can find a bounded sequence $\left\{g_{k}\right\} \subset C_{b}^{1}(H)$ converging to $g$ in $C_{b}(H)$. For each $k \in \mathbb{N}$ there exists a unique solution $\varphi_{\alpha, k}$ for the Hamilton-Jacobi problem

$$
\left(\lambda+\lambda_{0}\right) \varphi-L_{\alpha} \varphi+K_{r}(D \varphi)=g_{k}+\lambda_{0} \varphi(\lambda, g) .
$$

Then, since $g_{k}+\lambda_{0} \varphi(\lambda, g) \in C_{b}^{1}(H)$, as proved above, the corresponding closed loop equation has a unique solution $y_{\alpha, k}^{\star}(t)$. If we show that for any $T>0$ the sequence $\left\{y_{\alpha, k}^{\star}\right\}$ converges to some $y_{\alpha}^{\star}$ in $C([0, T] ; H), \mathbb{P}$-a.s. and in mean-square, then we easily have that $y_{\alpha}^{\star}$ is the solution of the closed loop equation (5.7).

For $k, h \in \mathbb{N}$ we define $v_{\alpha}^{k, h}(t)=y_{\alpha, k}^{\star}(t)-y_{\alpha, h}^{\star}(t)$. We have that $v_{\alpha}^{k, h}$ is the solution of the problem

$$
\begin{aligned}
\frac{d v}{d t}(t)= & A v(t)+F_{\alpha}\left(y_{\alpha, k}^{\star}(t)\right)-F_{\alpha}\left(y_{\alpha, h}^{\star}(t)\right)-D K\left(D \varphi_{\alpha, k}\left(y_{\alpha, k}^{\star}(t)\right)\right) \\
& +D K\left(D \varphi_{\alpha, h}\left(y_{\alpha, h}^{\star}(t)\right)\right), \quad v(0)=0 .
\end{aligned}
$$

By multiplying each side by $v_{\alpha}^{k, h}(t)$ and recalling (2.3) and (2.4), we have

$$
\begin{aligned}
& \frac{1}{2} \frac{d}{d t}\left|v_{\alpha}^{k, h}(t)\right|_{H}^{2} \leq c\left|v_{\alpha}^{k, h}(t)\right|_{H}^{2} \\
& +\left|D K\left(D \varphi_{\alpha, k}\left(y_{\alpha, k}^{\star}(t)\right)\right)-D K\left(D \varphi_{\alpha, h}\left(y_{\alpha, h}^{\star}(t)\right)\right)\right|_{H}\left|v_{\alpha}^{k, h}(t)\right|_{H} .
\end{aligned}
$$

Since $D K$ is locally Lipschitz continuous and according to Lemma 4.11 we have

$$
\sup _{k \in \mathbb{N}}\left\|D \varphi_{\alpha, k}\right\|_{0} \leq \sup _{k \in \mathbb{N}}\left(\left\|D g_{k}\right\|_{0}+\lambda_{0}\|D \varphi\|_{0}\right)<\infty
$$

we obtain

$$
\begin{aligned}
& \left|D K\left(D \varphi_{\alpha, k}\left(y_{\alpha, k}^{\star}(t)\right)\right)-D K\left(D \varphi_{\alpha, h}\left(y_{\alpha, h}^{\star}(t)\right)\right)\right|_{H} \\
& \leq c\left|D \varphi_{\alpha, k}\left(y_{\alpha, k}^{\star}(t)\right)-D \varphi_{\alpha, h}\left(y_{\alpha, h}^{\star}(t)\right)\right|_{H} .
\end{aligned}
$$

For each $x, y \in H$ we have

$$
\left|D \varphi_{\alpha, k}(x)-D \varphi_{\alpha, k}(y)\right|_{H} \leq c\left\|\varphi_{\alpha, k}\right\|_{2}|x-y|_{H},
$$

and then, since the sequence $\left\{g_{k}\right\}$ is bounded in $C_{b}^{1}(H)$, from (4.16) it follows that

$$
\left|D \varphi_{\alpha, k}\left(y_{\alpha, k}^{\star}(t)\right)-D \varphi_{\alpha, k}\left(y_{\alpha, h}^{\star}(t)\right)\right|_{H} \leq c_{\alpha}\left|v_{\alpha}^{k, h}(t)\right|_{H} .
$$


Moreover, if $\lambda_{0}$ is large enough, due to (5.5) we have

$$
\left\|D \varphi_{\alpha, k}-D \varphi_{\alpha, h}\right\|_{0} \leq c_{\alpha}\left\|g_{k}-g_{h}\right\|_{0} .
$$

Therefore, we can conclude that

$$
\left|D K\left(D \varphi_{\alpha, k}\left(y_{\alpha, k}^{\star}(t)\right)\right)-D K\left(D \varphi_{\alpha, h}\left(y_{\alpha, h}^{\star}(t)\right)\right)\right|_{H} \leq c_{\alpha}\left|v_{\alpha}^{k, h}(t)\right|_{H}+c_{\alpha}\left\|g_{k}-g_{h}\right\|_{0},
$$

so that from the Young inequality we have

$$
\frac{1}{2} \frac{d}{d t}\left|v_{\alpha}^{k, h}(t)\right|_{H}^{2} \leq c_{\alpha}\left|v_{\alpha}^{k, h}(t)\right|_{H}^{2}+c_{\alpha}\left\|g_{k}-g_{h}\right\|_{0}^{2} .
$$

By the Gronwall lemma this yields

$$
\sup _{t \in[0, T]}\left|y_{\alpha, k}^{\star}(t)-y_{\alpha, h}^{\star}(t)\right|_{H} \leq c_{T}\left\|g_{k}-g_{h}\right\|_{0}, \quad \mathbb{P} \text {-a.s. }
$$

for some constant $C_{T}$. Thus $y_{\alpha, k}^{\star}$ converges to some $y_{\alpha}^{\star}$ in $C([0, T] ; H), \mathbb{P}$-a.s and in mean-square, and it is not difficult to check that $y_{\alpha}^{\star}$ is the solution of the closed loop equation corresponding to the datum $g$.

By proceeding as in [9, Theorem 7.3] it is possible to show that when the space dimension $d$ equals 1 , under suitable assumptions there exist an optimal control and the corresponding optimal state.

TheORem 5.4. Assume that the space dimension d equals 1.

1. If the constant $m$ in Hypothesis 1 is less than or equal to 1 , then there exists a unique optimal control for the minimizing problem associated with the functional J. Furthermore, the optimal control $z^{\star}$ is related to the corresponding optimal state $y^{\star}$ by the feedback formula

$$
z^{\star}(t)=-D K\left[D V\left(y^{\star}(t)\right)\right], \quad t \in[0, T] .
$$

2. If DK can be extended as a Lipschitz continuous mapping from $E^{\star}$ into itself, then the same conclusion holds for any $x \in E$.

\section{REFERENCES}

[1] V. Barbu and G. Da Prato, Hamilton-Jacobi Equations in Hilbert Spaces, Research Notes in Mathematics 86, Pitman, Boston, 1983.

[2] P. Cannarsa and G. Da Prato, Second-order Hamilton-Jacobi equations in infinite dimensions, SIAM J. Control Optim., 29 (1991), pp. 474-492.

[3] P. Cannarsa and G. Da Prato, Direct solution of a second-order Hamilton-Jacobi equation in Hilbert spaces, in Stochastic Partial Differential Equations and Applications, Pitman Res. Notes Math. Ser. 268, G. Da Prato and L. Tubaro, eds., Longman, Harlow, UK, 1992, pp. $72-85$.

[4] S. Cerrai, A Hille Yosida theorem for weakly continuous semigroups, Semigroup Forum, 49 (1994), pp. 349-367.

[5] S. CERRAI, Elliptic and parabolic equations in $\mathbb{R}^{n}$ with coefficients having polynomial growth, Comm. Partial Differential Equations, 21 (1996), pp. 281-317.

[6] S. CERRAI, Differentiability with respect to initial datum for solutions of SPDE'S with no Fréchet differentiable drift term, Comm. Appl. Anal., 2 (1998), pp. 249-270.

[7] S. CERRAI, Smoothing properties of transition semigroups relative to SDE's with values in Banach spaces, Probab. Theory Related Fields, 113 (1999), pp. 85-114.

[8] S. CERRAI, Differentiability of Markov semigroups for stochastic reaction-diffusion equations and applications to control, Stochastic Process. Appl., 83 (1999), pp. 15-37.

[9] S. CERRAI, Optimal control problems for stochastic reaction-diffusion systems with nonLipschitz coefficients, SIAM J. Control Optim., 39 (2001), pp. 1779-1816. 
[10] P. L. Chow and J. L. Menald, Infinite dimensional Hamilton-Jacobi equations in GaussSobolev spaces, J. Nonlinear Anal., 29 (1997), pp. 415-426.

[11] M. G. Crandall, H. Ishit, and P. L. Lions, User's guide to viscosity solutions of second order partial differential equations, Bull. Amer. Math. Soc. (N.S.), 27 (1992), pp. 1-67.

[12] G. Da Prato And A. Debussche, Control of the stochastic Burgers model of turbulence, SIAM J. Control Optim., 37 (1999), pp. 1123-1149.

[13] G. Da Prato and A. Debussche, Dynamic programming for the stochastic Burgers equation, Ann. Mat. Pura Appl. (4), 178 (2000), pp. 143-174.

[14] G. Da Prato and J. Zabczyk, Stochastic Equations in Infinite Dimensions, Cambridge University Press, Cambridge, UK, 1992.

[15] E. B. Davies, Heat Kernels and Spectral Theory, Cambridge University Press, Cambridge, UK, 1989.

[16] T. E. Duncan, B. Maslowski, and B. Pasik-Duncan, Ergodic boundary point control of stochastic semilinear systems, SIAM J. Control Optim., 36 (1998), pp. 1020-1047.

[17] W. H. Fleming And H. M. Soner, Controlled Markov Processes and Viscosity Solutions, Springer-Verlag, New York, 1993.

[18] M. Freiduin, Markov Processes and Differential Equations: Asymptotic Problems, Lectures Math. ETH Zürich, Birkhäuser-Verlag, Basel, 1996.

[19] B. Goldys and B. Maslowski, Ergodic control of semilinear stochastic equations and the Hamilton-Jacobi equation, J. Math. Anal. Appl., 234 (1999), pp. 592-631.

[20] F. GozzI, Regularity of solutions of a second order Hamilton-Jacobi equation and application to a control problem, Comm. Partial Differential Equations, 20 (1995), pp. 775-826.

[21] F. GozzI, Global regular solutions of second order Hamilton-Jacobi equations in Hilbert spaces with locally Lipschitz nonlinearities, J. Math. Anal. Appl., 198 (1996), pp. 399-443.

[22] F. Gozzi AND E. RouY, Regular solutions of second-order stationary Hamilton-Jacobi equations, J. Differential Equations, 130 (1996), pp. 201-234.

[23] T. Haverneanu, Existence for the dynamic programming equation of control diffusion processes in Hilbert spaces, Nonlinear Anal., 9 (1985), pp. 619-629.

[24] P. L. Lions, Viscosity solutions of fully nonlinear second order equations and optimal control in infinite dimensions I: The case of bounded stochastic evolutions, Acta Math., 161 (1998), pp. 243-278.

[25] P. L. LIONS, Viscosity solutions of fully nonlinear second order equations and optimal control in infinite dimensions II: Optimal control of Zakai's equation, in Stochastic Partial Differential Equations and Applications, Lecture Notes in Math. 1390, G. DaPrato and L. Tubaro, eds., Springer-Verlag, Berlin, 1989, pp. 147-170.

[26] P. L. Lions, Viscosity solutions of fully nonlinear second order equations and optimal control in infinite dimensions III: Uniqueness of viscosity solutions for general second order equations, J. Funct. Anal., 86 (1989), pp. 1-18.

[27] A. LunARdi, Analytic Semigroups and Optimal Regularity in Parabolic Problems, BirkäuserVerlag, Basel, 1995.

[28] A. SwIEch, Viscosity Solutions of Fully Nonlinear Partial Differential Equations with Unbounded Terms in Infinite Dimensions, Ph.D. thesis, University of California Santa Barbara, Santa Barbara, CA, 1993. 